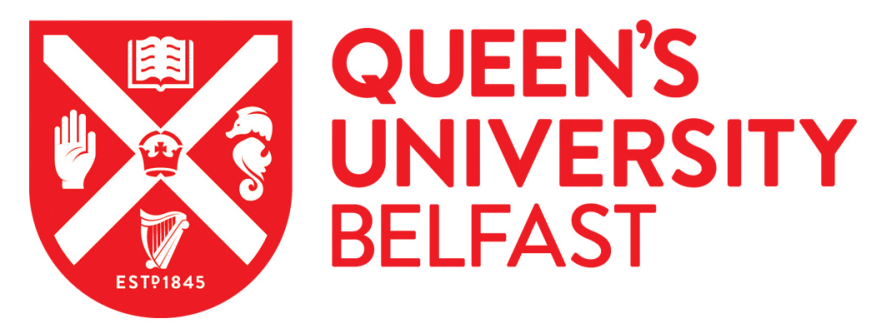

\title{
Multi-objective regression test selection in practice: An empirical study in the defense software industry
}

Garousi, V., Özkan, R., \& Betin-Can, A. (2018). Multi-objective regression test selection in practice: An empirical study in the defense software industry. Information and Software Technology, 103, 40-54.

https://doi.org/10.1016/j.infsof.2018.06.007

Published in:

Information and Software Technology

Document Version:

Peer reviewed version

Queen's University Belfast - Research Portal:

Link to publication record in Queen's University Belfast Research Portal

Publisher rights

(c) 2018 Elsevier B.V. All rights reserved.

This manuscript version is made available under the CC-BY-NC-ND 4.0 license http://creativecommons.org/licenses/by-nc-nd/4.0/,which permits distribution and reproduction for non-commercial purposes, provided the author and source are cited.

\section{General rights}

Copyright for the publications made accessible via the Queen's University Belfast Research Portal is retained by the author(s) and / or other copyright owners and it is a condition of accessing these publications that users recognise and abide by the legal requirements associated with these rights.

Take down policy

The Research Portal is Queen's institutional repository that provides access to Queen's research output. Every effort has been made to ensure that content in the Research Portal does not infringe any person's rights, or applicable UK laws. If you discover content in the Research Portal that you believe breaches copyright or violates any law, please contact openaccess@qub.ac.uk. 


\title{
Multi-objective regression test selection in practice: an empirical study in the defense software industry
}

\author{
Vahid Garousi \\ Information Technology Group \\ Wageningen University, \\ Netherlands \\ vahid.garousi@wur.nl
}

\author{
Ramazan Özkan \\ Consultant software engineer \\ Ankara, Turkey \\ rozkan0@gmail.com
}

\author{
Aysu Betin-Can \\ Informatics Institute, Middle East \\ Technical University, Ankara, Turkey \\ betincan@metu.edu.tr
}

\begin{abstract}
:
Context: Executing an entire regression test-suite after every code change is often costly in large software projects. To cope with this challenge, researchers have proposed various regression test-selection techniques.

Objective: This paper was motivated by a real industrial need to improve regression-testing practices in the context of a safety-critical industrial software in the defence domain in Turkey. To address our objective, we set up and conducted an "action-research" collaborative project between industry and academia.

Method: After a careful literature review, we selected a conceptual multi-objective regression-test selection framework (called MORTO) and adopted it to our industrial context by developing a custom-built genetic algorithm (GA) based on that conceptual framework. GA is able to provide full coverage of the affected (changed) requirements while considering multiple cost and benefit factors of regression testing. e.g., minimizing the number of test cases, and maximizing cumulative number of detected faults by each test suite.

Results: The empirical results of applying the approach on the Software Under Test (SUT) demonstrate that this approach yields a more efficient test suite (in terms of costs and benefits) compared to the old (manual) test-selection approach, used in the company, and another applicable approach chosen from the literature. With this new approach, regression selection process in the project under study is not ad-hoc anymore. Furthermore, we have been able to eliminate the subjectivity of regression testing and its dependency on expert opinions.

Conclusion: Since the proposed approach has been beneficial in saving the costs of regression testing, it is currently in active use in the company. We believe that other practitioners can apply our approach in their regression-testing contexts too, when applicable. Furthermore, this paper contributes to the body of evidence in regression testing by offering a success story of successful implementation and application of multi-objective regression testing in practice.
\end{abstract}

\section{Keywords:}

Regression testing; multi-objective optimization; genetic algorithms; empirical study; defence software industry; actionresearch 
This is a pre-print of the following paper that has been published in the Information and Software Technology (IST) journal: http://dx.doi.org/10.1016/j.infsof.2018.06.007

\section{TABLe OF Contents}

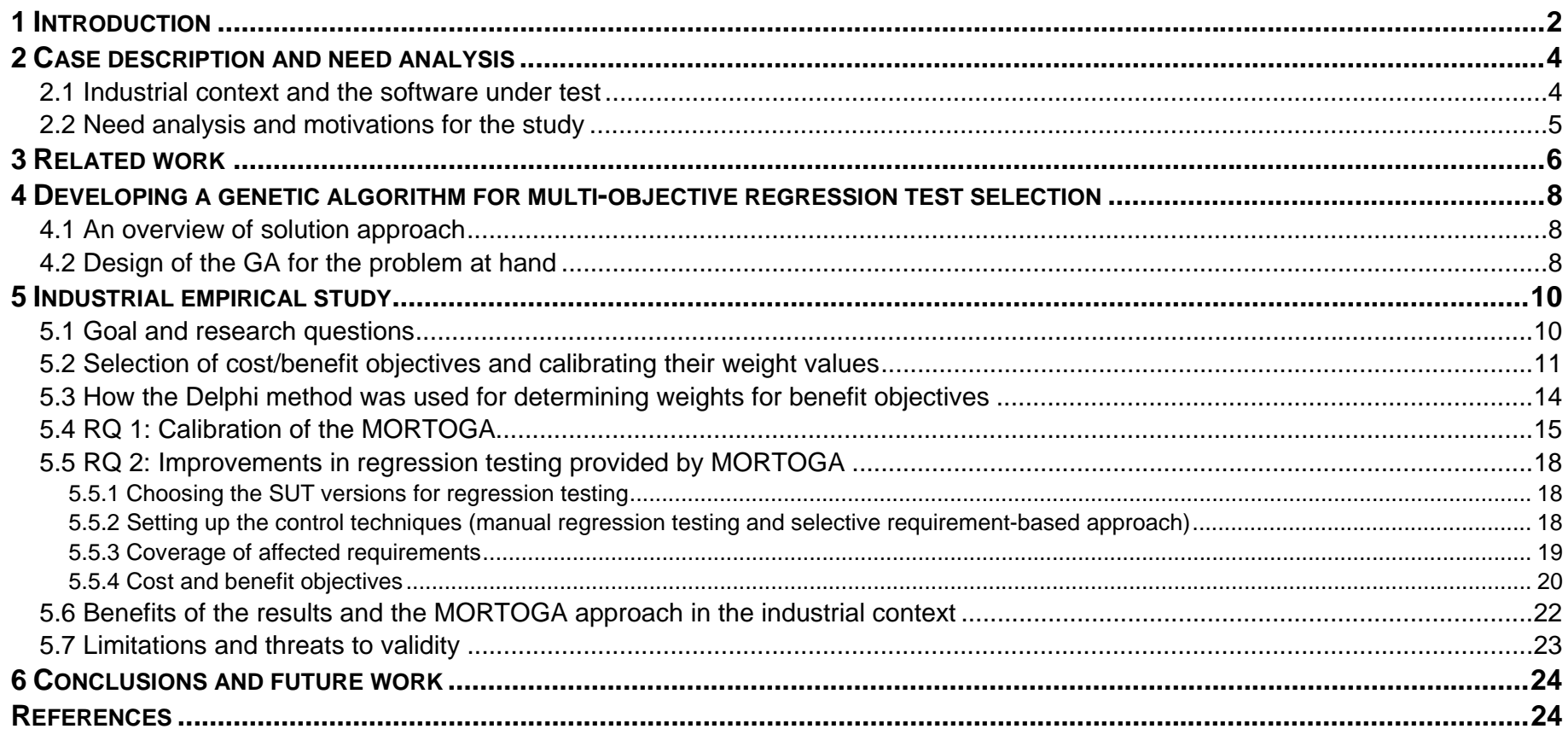

\section{INTRODUCTION}

Software testing is an important phase of the Software Development Life Cycle (SDLC). Furthermore, inadequate software testing could lead to major economic consequences, e.g., [1-3]. For example, a report called "Software fail watch" [1] published in 2017 by a large software company, Tricentis $\mathrm{GmbH}$, revealed that software failures caused $\$ 1.7$ trillion globally in financial losses in 2017 alone.

There are different types of software testing approaches. Regression testing is an important type of software testing activity, which is to test a Software Under Test (SUT) to ensure that code changes have not affected the existing functionality of the system [4]. The traditional and simplest approach to regression testing is to re-execute all the test cases that were used to test the software before the modifications were made, i.e., the "retest all" approach [4]. In practice however, re-executing all the previous test cases in regression testing after each software revision is not practical especially in the case of large software systems, due to time and budget constraints [5]. Regression testing is costly because, as software grows in size and complexity, a suite accumulate more tests and therefore take longer to run. For instance, a 2011 paper [6] reported that the regression (automated) testing of a Microsoft product required several days.

An alternative approach is selecting only a subset of the initial test suite. Such an approach is referred to as regression test-selection, test prioritization [7, 8], or test minimization [4]. Much research effort has been spent on finding costefficient methods for regression testing and many techniques have been proposed in the literature (refer to two survey papers in this area $[4,9])$. The techniques in this area are based either on single-objective optimization (e.g., reducing the number of test cases) or multi-objective optimization (e.g., minimizing the number of test cases and maximizing test coverage). For example, Harman [10] presented a conceptual framework for multi-objective regression test-selection, referred to as Multi-Objective Regression Test Optimization (MORTO), which considered a set of cost-based objectives 
This is a pre-print of the following paper that has been published in the Information and Software Technology (IST) journal: http://dx.doi.org/10.1016/j.infsof.2018.06.007

(such as test execution time and third-party costs) as well as a set of value-based objectives (such as code coverage and coverage of past fault).

The work reported in our paper is motivated by a real industrial need to improve regression-testing practices in the context of a safety-critical industrial software in the defence domain. Based on the industrial need, we set up and conducted an industry-academia collaborative [11, 12] project and followed the principles of "action-research" [13, 14]. After an extensive literature review, we chose the MORTO conceptual framework [10] as the baseline, and developed a custom-built genetic algorithm (GA) [15] to apply it in the project under study. In the subject project, before development and introduction of the new approach (as presented in this paper), regression test-selection was performed manually making it costly, subjective and error prone. Furthermore, quality of regression testing was highly dependent on the skills and experience of the test teams and test engineers. Therefore, there was a strong need for systematic approaches to regression test selection in the subject project. This need was especially very critical given the safety-critical nature of the Software Under Test (SUT) since it is a controller software system in the defence domain (further details not disclosed due to confidentiality).

Our regression test-selection project had several objectives: (1) to provide full coverage of the requirements that are affected by the program modifications, and (2) to improve the overall cost-benefit of the regression testing approach based on all the relevant regression selection criteria in the project, e.g., minimizing the number of test cases while ensuring full coverage of the affected requirement by regression test suites. Using the systematic guidelines for technology transfer provided by Gorschek et al. [16], as a team of researchers and practitioners, we jointly developed a search-based approach and transitioned this technology to the industrial context. Since the proposed approach has been beneficial in saving the costs of regression testing, it is now in active use in the company. After developing and implementing this systematic approach in the company, a positive outcome is that regression test-selection in the company is not ad-hoc anymore and we diminished the subjectivity and dependency of regression testing on experts' opinions.

The contribution of this paper is three-fold:

- Design and development of a multi-objective GA for multi-objective regression test optimization (i.e., our GAbased approach is an "instance" of the MORTO conceptual framework [10] for our specific context);

- Empirical calibration (tuning) of the GA (parameters such as GA population size, crossover and mutation rates) to ensure its best performance [17, 18] in our context; and

- Empirical evaluation of the proposed GA and comparing the efficiency of regression test-selection using the GA with the old (manual) test-selection approach, and another applicable approach that we found in the literature [19].

We have structured the rest of this paper as follows. We describe the project context, SUT and the needs/motivations for our work in Section 2. We review the related work in Section 3. Section 4 discusses how we developed the GA for multiobjective regression test selection in our context. Section 5 explains the application and empirical study of the approach in our industrial project and evaluates its effectiveness. Finally, in Section 6, we draw conclusions, and discuss our ongoing and future works.

Note that this paper summarizes the results of MSc thesis of the second author [20]. For the brevity of presentation and due to space limitations, we have summarized the approach and the empirical results in this paper. For more details, the reader can refer to the online version of the MSc thesis [20]. 
This is a pre-print of the following paper that has been published in the Information and Software Technology (IST) journal:

\section{CASE DESCRIPTION AND NEED ANALYSIS}

As discussed in Section 1, this work was initiated by an industrial need and we followed the principles of "actionresearch" [13, 14]. Therefore, we first review the industrial context and then discuss the needs and motivations for the study.

\subsection{INDUSTRIAL CONTEXT AND THE SOFTWARE UNDER TEST}

The subject project, SUT, is a safety-critical industrial controller software in the defence domain. The functionality of the SUT is defined by 935 system-level requirements. To verify those system-level requirements, the test team has developed 54 different test suites, each containing a set of test cases (test steps). The numbers of test steps in each of the test suites vary from 182 to 3,588 test cases. In total, there were 30,834 test cases (test steps) for the entire system. All black-box testexecution activities in this project are all done manually since the project has not yet adopted automated testing practices [21]. Manual execution times of each of the 54 test suites vary between 2 and 71 hours, per test suite, which results in a total of more than 1,500 hours of test execution labor. Considering that each workday has 8 hours, this translated to 187+ staff-days of manual test execution effort, for each single re-execution of the entire test suites. This denotes that the test team had to spend a large amount of time and effort on manual regression testing of the SUT. There were about 25 fulltime manual testers in the company assigned to run such large number of test cases in each round of regression testing on each version of the SUT.

The customer of the project is a major government organization in Turkey. At the time when the current industryacademia collaborative project started, the subject project had entered its acceptance-testing phase. The subject project is still ongoing as of this writing and the software is in the maintenance phase. A test group in the customer organization was tasked with executing acceptance tests and this group is authorized to determine the regression test coverage of each software build. The second author of this paper was a member of this test group for several years.

The project contractor is a large company with expertise in a broad range of products in defense. For confidentiality reasons, we only share limited details about the context and the subject project throughout this paper. The contractor company has achieved the CMMI Level 5 rating in the following areas: (1) software and systems engineering, (2) integrated product and process development, and (3) supplier sourcing. Because of having CMMI Level 5 rating, all software-testing activities are well documented and conducted in high standards by the project contractor.

For the subject project (SUT), there is an independent test group in the contractor company. The test group itself consists of several test teams, partitioned by the type of subsystems in the SUT. All of the test activities, conducted together with participation of the customer test team, are black-box testing, since the customer does not have access to the SUT's source code. As of this writing, as discussed above, the SUT is in the software maintenance phase and test teams conducted regression tests after each revision of the SUT with participation of the contractor and customer test teams.

Since the contractor company had achieved the CMMI Level 5 rating, it has a systematic metric/measurement program and a set of measurement databases in place. One of those measurement databases is used by the test team to store, in fine granularity, historical cost (effort) data for different cost factors in testing, e.g., costs of third-party services (in staff-hours) needed for a given test case, and system setup costs needed for a given test case. The cost (effort) database had historical going back several years at the time of the project reported in this paper. The teams regularly used such data to make various decisions w.r.t. test and test management in the company. During our empirical study reported in this paper, we also used some data from the cost (effort) database as reported in Section 5.2.

In case of a test failure, which is determined by the contractor, a software fix is implemented and a new software build is submitted. Before the introduction of improvements proposed in this paper, in order to assure the customer that software changes did not affect previously-verified functionalities, the contractor test team used to provide a list of possibly- 
This is a pre-print of the following paper that has been published in the Information and Software Technology (IST) journal: http://dx.doi.org/10.1016/j.infsof.2018.06.007

affected low-level requirements and proposed a regression test suite based on their analysis and experience for each software build. In the rest of the paper, we will refer to the set of test suites selected for regression as "regression test-set".

Contractually, in the subject project, a test suite cannot be divided in sub-parts (test cases or test steps) in the regression selection process. Therefore, a test suite is either executed in its entirety or not at all. Such a condition is due to various reason, one being the need for systematic "end-to-end"" testing [22], in which a black-box test suite tests a complete endto-end behavioral scenario of the SUT, e.g., one use-case of the safety-critical controller software.

\subsection{NEED ANALYSIS AND MOTIVATIONS FOR THE STUDY}

In the project under study, in the past, each time when a software fix was implemented, the customer test team used to first evaluate the list of affected system requirements and the regression test-set proposed by the contractor test team. The customer test team would then either accept the proposed regression test-set or would request adding more test suites to it. The entire approach was ad-hoc, subjective and purely based on expert opinion. To make the matters worse, there was an inherent challenge (conflict) in the process since the contractor test team always tried eagerly to "minimize" the scope and size of regression test-set, naturally to lower their own test cost and effort, the customer test team usually had the tendency to "enlarge" the scope of regression test-set as much as possible, by adding extra test suites "to be on the safe side". As a result, the regression testing process needed extensive meetings and discussions about the extent (scope) of the regression test-set. This unsystematic process usually led to long, controversial (and often unpleasant) discussions and at the end of negotiations, there was a test suite which made neither side happy. Ironically, as per our analysis, the resulting test suites usually did not even achieve 100\% coverage of the affected (changed) requirements, since they were derived with ad-hoc mechanisms, e.g., ad-hoc and complex Excel-sheet- and heuristics-based decision-support.

Neither the customer nor the contractor test team had access to source code and lacked detailed information about the software (the entire software is highly classified due to its nature), and therefore, decisions about the scope of regressing testing were made subjectively and mostly based on rules of thumb.

After several rounds of meetings among the both test teams and the research team (authors of this paper), both test teams mentioned that they were keen to improve the existing ad-hoc regression test-selection practices and establish a systematic, more effective and efficient regression test-selection approach. The important criteria that the stakeholders raised for regression testing were to include a set of cost and value (benefit) factors (as discussed next). Let us clarify that the project stakeholders were typical and included: the customer, developers, testers and managers. We were asked to develop a better regression testing approach that would cover all the affected system requirements, by a new software revision (maintenance activity), while, at the same time, would entail the least cost and provide maximum value (benefit) for regression testing.

The stakeholders observed in the past that multiple cost and benefit factors were associated with regression testing of the subject system. Two example cost factors that we identified in discussions with the stakeholders are: (1) Test execution time: time required for executing a test suite (conducted mostly manually in the project as test automation is not widely practiced in this project); and (2) Third party cost: the cost of services procured from third party organizations, since some of the test suites in the subject system required specific services and hardware devices that cannot be provided by the internal stakeholders and had to be acquired/rented from third party organizations (often, due to their "classified" nature).

Similarly, we identified the following example value (benefit) factors in our discussions with the stakeholders: (1) Number of detected faults: experience has shown that as the software is developed and periodically upgraded, the phenomenon of re-emergence of similar defects is quite common [23]. Thus, the detection ability of past faults by a given regression test suite is seen as one of its values and it makes sense, in regression testing, to give priority to test suites which detect higher number of past faults; (2) Faults severity: it is not only the number of faults detected by a test suite 
This is a pre-print of the following paper that has been published in the Information and Software Technology (IST) journal: http://dx.doi.org/10.1016/j.infsof.2018.06.007

that is important, but also the severity of faults. Test suites which detect more severe faults are better to be prioritized in regression testing.

While some test suites may be deemed of high priority in terms of some of the above cost objectives, they may be assessed in lower priority in terms of the value (benefit) objectives. Also, similar to the many other contexts in the optimization research literature, the cost and value factors in this context represented "conflicting" objectives and both categories of objectives should be considered holistically in the optimization approach. As a result, this turns the problem into a challenging multi-object optimization problem [24]. Thus, the work reported in this paper was initiated to develop a solution for the problem at hand.

\section{RELATED WORK}

Using an existing 7-step process for technology transfer, proposed by Gorschek et al. [16], after identifying the need and motivations for the study (as discussed in Section 2), we conducted a careful literature review to be aware of the state of the art and practice in this area, and possibly find a suitable approach as the baseline to be adopted/extended in our project.

(Automated) regression test-selection has become a research field of its own since a few decades ago and a large number of regression test-selection techniques have been presented in this area (perhaps at least 200 studies so far according to [4]). Given such a large number of papers, it is not practical to review all of them in this section, but instead, we review a few of the many survey (review) papers that have been published in this area $[4,9,25-28]$, as listed in Table 1. These review papers were identified in a recent systematic literature review (SLR) of literature reviews in software testing [29] (a so-called "tertiary" study).

As listed in Table 1, we can see the intensive focus of the research community on regression test minimization, selection and prioritization. Some of these review papers have focused on more focused topics, e.g., application of genetic algorithms for test-case prioritization in [27], and have reviewed a smaller number of (primary) studies (7 papers in [27]). However, other review papers in this set have taken a "broader" focus and have reviewed a larger pool of papers in this area (189 papers in [4]). Interested readers can use any of the review papers listed in Table 1 as an "index" to the vast body of knowledge in this area and study any of the many papers in this area.

The concept of regression test-selection is closely related to two similar concepts: test-case prioritization and test-suite minimization [4]. While test-case selection seeks to identify the test cases that are relevant to some set of recent changes in the system under test (SUT), test-suite minimization seeks to eliminate redundant test cases in order to reduce the number of tests to run. Test-case prioritization seeks to order test cases in such a way that early fault detection is maximized. Those techniques sort (prioritize) test cases selected using a modification-based technique according to coverage or other objectives [30].

Table 1- A summary of the existing review (survey) papers in the field of regression test-selection (by years of publication)

\begin{tabular}{|c|c|l|c|c|}
\hline $\begin{array}{c}\text { Year of } \\
\text { publication }\end{array}$ & Reference & Paper title & $\begin{array}{c}\text { Type of survey } \\
\text { (review) paper }\end{array}$ & $\begin{array}{c}\text { Num. of (primary) } \\
\text { studies reviewed }\end{array}$ \\
\hline 2010 & {$[9]$} & $\begin{array}{l}\text { A systematic review on regression test selection } \\
\text { techniques }\end{array}$ & SLR & 27 \\
\hline 2011 & {$[25]$} & Regression test selection techniques: a survey & Regular survey & 118 \\
\hline 2012 & {$[4]$} & $\begin{array}{l}\text { Regression testing minimization, selection and } \\
\text { prioritisation: a survey }\end{array}$ & Regular survey & 189 \\
\hline 2012 & {$[26]$} & $\begin{array}{l}\text { Systematic literature review on regression test } \\
\text { prioritization technique }\end{array}$ & SLR & 65 \\
\hline 2012 & {$[27]$} & On the application of genetic algorithms for test- & SLR & 7 \\
\hline
\end{tabular}


This is a pre-print of the following paper that has been published in the Information and Software Technology (IST) journal: http://dx.doi.org/10.1016/j.infsof.2018.06.007

\begin{tabular}{|c|c|l|c|c|}
\hline & & case prioritization: a systematic literature review & & 120 \\
\hline 2013 & {$[28]$} & $\begin{array}{l}\text { Test-case prioritization: a systematic mapping } \\
\text { study }\end{array}$ & Systematic mapping & SLR \\
\hline 2018 & {$[27]$} & $\begin{array}{l}\text { Test-case prioritization approaches in regression } \\
\text { testing: a systematic literature review }\end{array}$ & 69 \\
\hline
\end{tabular}

Some of regression test-selection techniques work by comparing outputs produced by old and new versions of the SUT. In these techniques, tests that will cause the modified program to produce different output than the original program are selected. Most of these techniques focused on software modifications in the SUT by using source-code analysis methods such as execution trace analysis, data-flow analysis and control-flow analysis [31-38]. These techniques were not applicable in our case since we had no access to source code.

Test-selection based on test-suite minimization aim at finding a smaller group of test cases having the same coverage with respect to produced outputs of the old and modified SUT. For instance, Gu et al. [19] developed an algorithm that minimizes test suites by maximizing the coverage of the changed requirements (i.e. the requirements that are affected by the program modifications) and minimizing coverage of unaffected (irrelevant) requirement.

By reviewing the literature, we also realized that the majority of existing regression test-selection techniques have focused on a single objective or two objectives. However, in many real-world regression testing scenarios, including our project as discussed in Section 2.2, we have observed that there are more than two objectives (criteria) that have an impact on effectiveness and efficiency of a regression test suite such as execution time, coverage, priority (assigned by experts), and fault history. Therefore, industrial applicability of many of the existing single- or dual-objective regression test-selection techniques is limited [4]. To tackle the regression testing problem in such contexts, Harman discussed the need for multiple criteria by coining the term Multi-Objective Regression Test Optimization (MORTO) and provided a list of possible regression test-selection criteria in [10]. As presented in [10], MORTO is not a fully-developed approach yet, and only serves as a conceptual framework for this problem.

We further reviewed the literature regarding existing multi-objective regression test optimization approaches, and we list the approaches that we have found in Table 2. We also provide in Table 2 the list of cost/benefit objectives considered in each approach. The MORTO conceptual framework [10] seems the most comprehensive approach in terms of number of support cost/benefit objectives. After our careful literature review, we selected the MORTO approach [10] as our base framework, since it defines a consolidated framework supporting multiple objectives for regression testing, and compared to the other approaches, e.g. [6], its conceptual framework of cost and benefit drivers was more applicable to our context and the set of our cost and benefit drivers (as discussed in Section 2.2).

As we can see in in Table 2, some of the identified approaches considered code coverage as a value objective which can only be utilized when conducting white-box (regression) test selection. To the best of our knowledge, the work of Gu et al. [19] is the only regression test approach which has considered black-box objectives, i.e., coverage of affected requirements, and coverage of unaffected (irrelevant) requirements. Since in our study context, we did not have access to the system source code, only a black-box (regression) test selection was feasible. Thus, from the set of all existing approaches in the literature, only the approach of $\mathrm{Gu}$ et al. [19] could be applied in our context which we did (details in Section 5.4).

The other category of related work is the group of studies reported for testing software systems in the "defense" domain. While most of the efforts in this area are "classified" (confidential) and are not usually reported in publication venues (i.e., journals and conferences), some researchers and practitioners have reported studies in this area, e.g., [39-45]. “CrossTalk: The Journal of Defense Software" is a dedicate journal for software engineering on the defense domain and some of its papers are on testing, e.g., $[42,43]$. There are also sources in the grey literature discussing software testing in the defense domain, e.g., [46-49]. However, we did not find any studies, neither in formal or grey literature, in the topic of regression test optimization for defense-related software systems. 
This is a pre-print of the following paper that has been published in the Information and Software Technology (IST) journal:

http://dx.doi.org/10.1016/j.infsof.2018.06.007

Last but not the least, the focus of regression test approach in this work is to minimize the cost of regression testing while maximizing its benefits. Thus, in a broader sense, this work relates to the body of knowledge in Value-based Software Engineering (VBSE) [50] and software-engineering economics [51].

Table 2- A review of the existing multi-objective regression test optimization approaches

\begin{tabular}{|c|l|l|l|}
\hline Reference & Year & Cost objectives & Benefit (value) objectives \\
\hline$[52]$ & 2007 & Execution time & Code coverage, fault history \\
\hline$[19]$ & 2010 & - & $\begin{array}{l}\text { Coverage of affected requirements, coverage of } \\
\text { unaffected (irrelevant) requirements }\end{array}$ \\
\hline$[10]$ & 2011 & $\begin{array}{l}\text { Execution time, data access costs, third } \\
\text { party costs, technical resources costs, setup } \\
\text { costs, simulation costs }\end{array}$ & $\begin{array}{l}\text { Code based coverage, non-code-based coverage, fault } \\
\text { model sensitive, fault history sensitive, human } \\
\text { (tester) preferences, business priority }\end{array}$ \\
\hline$[53]$ & 2012 & Computational cost of tests & Statement coverage \\
\hline$[54]$ & 2014 & Execution time & Code coverage \\
\hline$[55]$ & 2015 & & $\begin{array}{l}\text { Average percentage of coverage achieved, average } \\
\text { percentage of coverage of changed code, average } \\
\text { percentage of past fault coverage }\end{array}$ \\
\hline$[56]$ & 2015 & Execution time & Code coverage, code diversity \\
\hline
\end{tabular}

\section{DEVELOPING A GENETIC ALGORITHM FOR MULTI-OBJECTIVE REGRESSION TEST SELECTION}

We first provide an overview of our solution approach and provide justification of why we choose genetic algorithms (GA) as the solution approach. We then present how we design a GA based on MORTO [10].

\subsection{AN OVERVIEW OF SOLUTION APPROACH}

The MORTO conceptual framework [10] proposes resolving the regression test-selection problem as a multi-objective optimization problem. Multi-objective optimization means optimizing more than one objective simultaneously [57]. NPhardness challenge of multi-objective optimization makes heuristic search algorithms the only viable option [58]. Heuristic-based optimization algorithms may not produce optimal solutions but they produce a solution in reasonable time.

Several heuristic search algorithms are widely used in the optimization literature and also in Search-based Software Engineering (SBSE) [59], e.g., simulated annealing, Tabu search, ant colony and genetic algorithms (GA) [60]. Because of its scalability and flexibility [18], and because it has been widely used in SBSE [59], we decided to use a GA-based approach to formulate and solve the multi-objective regression test optimization problem at hand. In two previous SBSE problems in other areas of software engineering [18, 61], we had also developed and used GA-based approaches.

\subsection{DESIGN OF THE GA FOR THE PROBLEM AT HAND}

Genetic algorithms (GA) are a heuristic search-based optimization method that mimics the process of natural selection [15]. In a GA, a gene represents an independent variable in the optimization problem and, as in nature, each chromosome which is a set of genes represents a (candidate) solution to the problem at hand.

To solve our problem at hand, we designed a GA in which each gene represents a test suite to be used in regression testing, and each chromosome represents a set of test suites (i.e., a regression test-set). To represent genes, we used the binary representation, in which each binary variable (gene) corresponds to a test suite. For each binary variable (bit), the value of " 1 " means existence of the given test suite in the solution (regression test-set), and " 0 " means its absence. Each "individual" in the population corresponds to a regression test-set and is represented as an array of size $\mathrm{M}$ where $\mathrm{M}$ is the 
This is a pre-print of the following paper that has been published in the Information and Software Technology (IST) journal: http://dx.doi.org/10.1016/j.infsof.2018.06.007

number of all regression test suites for a given SUT. We show in Figure 1 a visual illustration of the gene/chromosome representation in our GA, which we refer to as MORTOGA in the rest of this paper. In this example, number of genes in a single chromosome $(M)=54$ which is the number of test suites for the SUT used in this study (as discussed in Section 2.2).

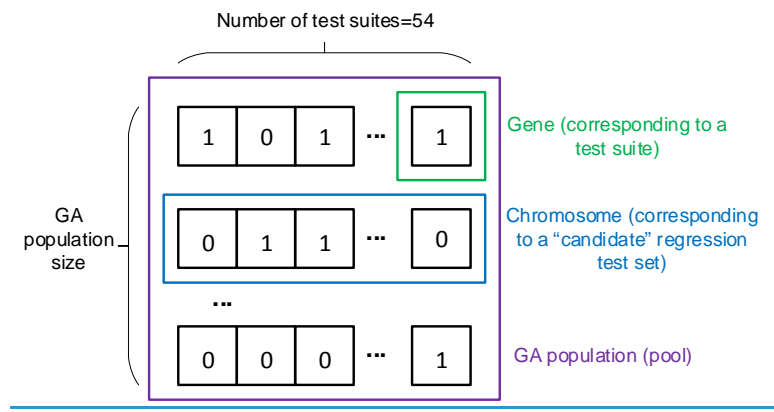

Figure 1- Representation of GA chromosomes in MORTOGA

The GA takes as input the set of all regression test suites and will select a subset of them, via the above chromosome structure, which yields the highest benefit and lowest cost for regression testing.

When designing the GA for the problem at hand, we paid special attention to three aspects: coverage of affected requirements, minimization of regression testing costs, and maximization of benefits. The first aspect guarantees that every candidate solution covers all of the affected requirements (i.e. the requirements that are affected by the program modifications). This part is realized by a constraint function in the GA which uses a given requirement-traceability matrix [62] to consider the relationships between test suites and requirements. This traceability matrix is indeed the core structure of our approach to ensure the full coverage of affected requirements. The matrix also makes the complex relationship between test suites and requirements understandable and usable in the regression test-selection process. The constraint function is applied whenever any GA individual (candidate solution) in the population is created and is changed, i.e.., when generating the initial population, and also after applying the GA's crossover and mutation operators [15]. Similar to the usual practice when designing GAs in SBSE, e.g., [18, 60, 61], any GA individual that does not pass the constraint function is discarded from the GA pool (population).

Since our GA is multi-objective (to consider both cost and benefit factors of regression testing), we had the choice of pursuing either of the two standard approaches [63]: (1) "Pareto" optimality to really consider both objectives, or (2) scalarization [64] which would require converting the multi-objective problem into a single-objective problem. Most of the existing multi-objective regression test selection techniques using Pareto optimality have used optimization algorithms such as NSGA-II [65] or the DIV-GA (DIVersity-based Genetic Algorithm) [53].

In approaches based on Pareto optimality, users should do the task of selecting a single solution from a large Paretooptimal. In discussions with stakeholders (decision makers) in our project, the Pareto optimality approach was not seen favorable since it would have complicated the regression solution approach for the team. If we had decided to proceed with the Pareto optimality approach, the team would have to organize extra meetings to subjectively choose one of the available options in the Pareto optimal set, provided by the GA. Furthermore, since the number of cost and benefit factors are more than three (actually nine) in our case (to be discussed in Section 5.2), visualization of the optimal solution set on the Pareto front to choose one optimal result would have been quite challenging to be implemented in our context. In fact, the stakeholders felt "overwhelmed" by us discussing the idea of having to choose one optimal solution from a large set of candidate solution on the Pareto front. Thus, proceeding via the route of multi-objective optimization using approaches such as NSGA-II was not a feasible nor a suitable approach in our context. On the other hand, we found that the scalarization- (weighted sum-) solution approach [64] is popular in the operations-research community, e.g., [64, 66, 67], and would provide a practical, easy-to-use and computationally-efficient approach. For the reasons discussed above, we 
This is a pre-print of the following paper that has been published in the Information and Software Technology (IST) journal: http://dx.doi.org/10.1016/j.infsof.2018.06.007

chose the scalarization approach and converted the multi-objective problem to a single-objective problem as discussed next.

To implement multi-objective optimization as a scalarized single-objective GA, we defined a fitness (objective) function for our GA. Using this fitness function, the GA would find the best regression test-set among the candidates. We show in Equation 1 the fitness function, in which the cost and value objectives are combined into one single objective by using a weighting scheme. The fitness (objective) function includes the weighting factors (coefficients) and (cost/benefit) objectives. The actual weighting values to be used inside Equation 1 would be specific for a given subject project (SUT). For our specific case-study SUT, we will discuss in Section 5.2 how we determined the values for the weighting values in the fitness function.

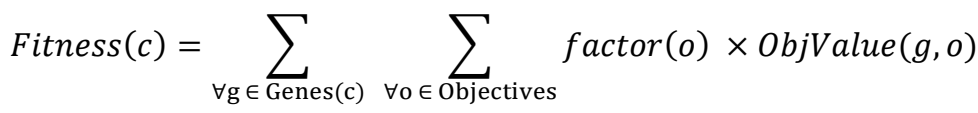

\section{Equation 1 - Fitness (objective) function of the GA}

In Equation 1, Fitness $(c)$ represents the fitness function value for a given chromosome $c$. The term ObjValue $(g, o)$ is the value of each objective (cost or benefit objectives) for a given test suite (gene) g. The term factor(o) denotes the predetermined weight (ratio) of each objective $o$ in the overall fitness value.

To consider cost and benefit objectives properly, the factor coefficients of benefit objectives are positive values while the factor coefficients of cost objectives are negative values, and the goal is to maximize the overall fitness value.

Note that the GA has been developed in an extensible manner for the objectives, any set (combination) of cost and benefit objectives can be plugged into the fitness function, depending on the SUT. We implemented our GA in Matlab and its code is available open-source online [68]. In the rest of this paper, we refer to our approach and GA as MORTOGA.

\section{INDUSTRIAL EMPIRICAL STUDY}

Once we designed the MORTOGA approach, which was based on our industrial need (Section 2), we applied it to our industrial setting in an empirical study to assess its usefulness. We discuss the empirical study's goal, research questions (RQs) and results next.

\subsection{GOAL AND RESEARCH QUESTIONS}

The goal of the empirical study was to improve the current manual regression test-selection process of a large industrial safety-critical software by applying the MORTOGA approach, as developed in Section 4. Before applying the GA on the problem domain (SUT), we were aware from the SBSE literature that the GA's internal parameters (e.g., population size, crossover and mutation rates) had to be first properly "tuned" (calibrated) empirically to ensure its best performance [17, 18]. Various studies, e.g., [17, 18], have shown that, when not calibrated properly, performance and outputs generated by SBSE techniques could be sub-par and far from the best outcomes. Thus, empirical calibration of MORTOGA's internal parameters (e.g., population size, crossover and mutation rates) to the problem at hand was our first task in the empirical study.

Based on the above goal, we posed two research questions (RQs):

- RQ 1: How can the MORTOGA be best calibrated (tuned) to yield the best results in the context under study? As discussed above, a GA's internal parameters (e.g., population size, crossover and mutation rates) have to be also properly tuned (calibrated) empirically to ensure its best performance [17, 18].

- RQ 2: How much improvement in regression testing does MORTOGA approach provide compared to the manual regression test-selection and the selected approach from the literature, the requirement coverage-based approach [19]? 
This is a pre-print of the following paper that has been published in the Information and Software Technology (IST) journal: http://dx.doi.org/10.1016/j.infsof.2018.06.007

RQs 1 and 2 will be addressed in Section 5.4 and 5.5. But before answering them, to adopt and apply the GA in the subject project, we had to first select the cost/benefit objectives in the project under study, and then calculate their weighting values to embed inside the fitness function in Equation 1, which we discuss next.

\subsection{SELECTION OF COST/BENEFIT OBJeCtiveS AND CALIBRATING THEIR WEIGHT VALUES}

As discussed in the MORTOGA design (Section 4.2), before using the GA approach in the project, we needed to calibrate the GA with cost and benefit weight values. We developed the GA in a flexible / extensible manner in terms of the objectives, i.e., various sets of cost and benefit objectives can be plugged into the fitness function, depending on the system and project under test.

As a result of meetings with various stakeholders in the project, we selected the following cost/benefit objectives for the project under test. While we explain each objective next, we also discuss how its weighting value was estimated:

1. Test execution time (of each test suite in): This is the time (and cost) required for executing a test suite. All test suites were manual tests as test automation was not practiced (implemented) in this project. We collected test execution time data from detailed test record sheets that include the date, time, and test engineers' names, as done in the project in the past. Some of the test suites were executed more than once in the past within the scope of regression testing as determined by the old test strategy and test process. For those test suites, their mean of execution times was calculated. Unit of test execution time is staff-hour (man-hour).

2. Third-party cost: Cost of services procured from third party organizations. In the SUT, some of the test suites require specific services (dependencies) that cannot be provided by the internal stakeholders and require services by third parties, e.g., setting up specific hardware systems which the SUT needed to execute. These costs were mostly in the context of Model-in-loop (MIL) and Hardware-in-loop (HIL) testing [69, 70] of the SUT, which is an embedded system. Third-party needs (dependencies) for each test suite were collected from prerequisites sections of test procedures, and were categorized as shown in Table 3. To systematically quantify the weights for different cost factors, we used historical cost (effort) data from the company's effort database (as discussed in Section 2.1) which had a long history of data for execution of test cases and actual costs associated with testing in the past several years for the SUT. Using historical effort and cost data from the past (analogy-based effort estimation) is a wide-spread approach in software effort estimation [71, 72], and test effort estimation, e.g., [73]. In the company's effort/cost database, effort data were stored in staff-hour. However, cost data were in monetary values (i.e., Turkish Lira), e.g., simulation-support as shown in Table 3were related to costs of external simulation systems (e.g., simulating certain scenarios in the defense domain) to enable Model-in-loop (MIL) and Hardware-in-loop (HIL) testing of the SUT. Such costs had fixed values per test suite and we converted their monetary values to the baseline (Test execution time in staff-hours) for all other cost factors as shown in Table 3, by converting monetary values to average cost of a test engineer for the company (salary and other costs). Two example items for third-party cost, as shown in Table 3 are simulation-support and satellite service costs. By calculating the mean (average) of cost (effort) data for these two items and rounded the values to the nearest integers, we calculated costs values as 2 and 40 staff-hour, respectively. As discussed above, all the costs were normalized into one cost factor (the baseline: Test execution time) and into one unit (staff-hours). This normalization ensures being able to combine all cost objectives in the fitness function (Equation 1).

3. System setup cost: Effort required for setup of system to execute the test suites. Some test suites required devices, services, files or a specific mode of the system. We also collected the costs related for such items from prerequisite sections of test procedures by test team. We found that system setup cost was divided into three types: (1) regular system setup, (2) special disk preparation, and (3) scenario generation setup, with weights as shown in Table 3. We determined weight values of system setup cost factors in a similar way to third-party cost, as discussed above, i.e., using historical cost (effort) data from the company's effort database. 
This is a pre-print of the following paper that has been published in the Information and Software Technology (IST) journal: http://dx.doi.org/10.1016/j.infsof.2018.06.007

4. Technical resources cost (test execution environment) to be spent by the internal team in the company: There are different test environments for the subject system, e.g., software on simulated hardware lab. Usage of these environments also incurs costs. We excluded the lab installation costs because installation of the lab was done only once. The other cost items that we included were: simulated software in the lab (i.e., Software-in-the-Loop, SIL), software on real hardware (HIL), real system or any combination of these environments. While usage of the simulated software in the lab (i.e., Software-in-the-Loop, SIL) testing approach had a cost of 4 staff-hours, testing the model of "All on the real system" had a cost of 48 staff-hours.

5. Verification (oracle) cost: Verification of specific requirement items in this context is not trivial and entails non-trivial costs. They usually require post-analysis of data collected during testing. We also collected the verification costs from the historical data related to how much time verification activities had taken.

Table 3- Different items of the cost objectives and their assigned weight values

\begin{tabular}{|c|c|c|}
\hline Cost groups & Items & $\begin{array}{c}\text { Weight } \\
\text { (Staff-hour } \\
\text { factor) }\end{array}$ \\
\hline Test execution time & Execution time of a test suite. This is the baseline for all other cost factors, thus its weight=1. & 1 \\
\hline \multirow{5}{*}{ Third-party costs } & $\begin{array}{l}\text { Simulation support costs: Costs of external simulation systems (e.g., simulating certain } \\
\text { scenarios in the defense domain) and services to enable Model-in-loop (MIL) and Hardware-in- } \\
\text { loop (HIL) testing }\end{array}$ & 2 \\
\hline & $\begin{array}{l}\text { Technical support costs: In some test activates, there was a need to get technical support to } \\
\text { install and troubleshoot certain hardware systems. }\end{array}$ & 8 \\
\hline & $\begin{array}{l}\text { Equipment service costs: Costs associated with renting (borrowing) hardware equipment to } \\
\text { enable testing }\end{array}$ & 12 \\
\hline & Flight service costs: Costs associated with & 32 \\
\hline & Satellite service costs: Costs associated with & 40 \\
\hline \multirow{3}{*}{ System setup costs } & Basic setup costs: To conduct testing, the SUT had to be set up (be prepared) for testing. & 2 \\
\hline & $\begin{array}{l}\text { Special disk preparation costs: To conduct testing, we needed to set up test data on a specific } \\
\text { disk storage of an embedded unit. }\end{array}$ & 8 \\
\hline & $\begin{array}{l}\text { Configuration generation and update costs: For regression testing, testers had to review, } \\
\text { regenerate and update the test configurations of the SUT and its various components, when } \\
\text { necessary. }\end{array}$ & 40 \\
\hline \multirow{5}{*}{$\begin{array}{l}\text { Technical resources } \\
\text { costs (internal in } \\
\text { the company) }\end{array}$} & Software-in-the-Loop (SIL) testing costs: To enable testing in the SIL mode & 4 \\
\hline & $\begin{array}{l}\text { Hardware-in-loop (HIL) + SIL testing costs: To enable testing in the combination of HIL+SIL } \\
\text { modes }\end{array}$ & 8 \\
\hline & $\begin{array}{l}\text { HIL + SIL testing + auxiliary lab software costs: A certain type of testing with HIL+SIL modes } \\
\text { and including auxiliary lab software }\end{array}$ & 12 \\
\hline & HIL + SIL + partial testing using the real system: Self explanatory & 32 \\
\hline & Testing fully done on the real system: Self explanatory & 48 \\
\hline Verification cost & Post-test analysis costs (e.g., test oracle) & 8 \\
\hline
\end{tabular}

On the other hand, in addition to the cost objectives, we also needed to identify the benefit objectives of regression testing. After meetings with stakeholders, we identified the following four benefit objectives, as discussed next, and shown in Table 4.

1. Cumulative number of detected faults by each test suite in the past: Experience has shown that as the software is developed and periodically updated, the phenomenon of re-emergence of similar defects is quite common [23]. Thus, the fault detection ability of a regression test suite is seen as a benefit (value) and it is important, in regression testing, to give priority to test suites which have detected more faults in the past. There have been existing studies on this topic, e.g., [74, 75]. We collected the data for this objective from the project's fault database. 
This is a pre-print of the following paper that has been published in the Information and Software Technology (IST) journal: http://dx.doi.org/10.1016/j.infsof.2018.06.007

2. Number of detected faults in the last version: In addition to the cumulative number of detected faults (the previous objective), the number of faults detected by a test suite in the last version provides awareness about the "recent" fault detection ability of a test suite. We saw cases when a test suite may not have had detected many faults in the past cumulatively, relative to other test suites, but has detected many faults just in the last version of the SUT. Because of this contribution, we added the number of detected faults in the last version as a separate value objective to the list of objectives. We also obtained the data for this objective from the fault database.

3. Faults severity: Not only the number of faults detected by a test suite is important, but also the severity of those faults. Test suites which detect more severe faults are better to be prioritized in regression testing [74]. In the project, three severity values were already defined in the faults database: 1 (most severe), 2 and 3 (least severe). For each test suite, its previously-detected faults were grouped based on priority level. Afterward, we applied the following formula, defined in decision-making meetings with consensus of stakeholders: Fault severity (a test suite)= $\left(3 * N u m \_f a u l t s \_s e v e r i t y \_1\right)+\left(2 * N u m \_f a u l t s \_s e v e r i t y \_2\right)+\left(1^{*} N u m \_f a u l t s \_s e v e r i t y \_3\right)$.

4. Test-suite execution priority: Based on some rationale which are hard (or impossible) to formulate (quantify), the test team may assign some sort of "Priority" to a regression test suite, on their experience, which denotes its importance. In our context, the test team assigned the priorities by using the Delphi method for each test suite based on their experience and their insight about the fault detection ability of each test suite. It is based on an ordinal metric and there are three priority levels: 1, 2 and 3, with the value 1 being the most important one.

Determining the weight values of benefit objectives was not as straightforward as the cost objectives. For cost objectives, we already had the historical cost data from which we could "quantify" cost and effort relatively easily, and were able to assign weight values for cost objectives. However, for benefit objectives such as: Cumulative number of detected faults and Faults severity, it was not immediately obvious how we could "quantify" benefits in the MORTOGA's formula (Equation 1). To tackle this need, by reviewing the literature, we used the wideband Delphi method [76] to gather experts' opinions to determine the importance of and to quantify benefits. To keep the focus of this section on cost/benefit objectives, we separate the discussion on details of the operational execution of the Delphi method and present that aspect in Section 5.3.

As discussed in Section 4.2, since the MORTOGA is multi-objective (to consider both cost and benefit factors), we used the scalarization approach [63] to fit all the objectives into one single objective function. In order to provide the intended weight values for all the objectives in the fitness value calculations, all the objectives were scaled (normalized) to the range of $[0,1]$ by using the following formula: scaledValue =Value/MaxValue.

As shown in Table 4, all the cost objectives were given the equal negative weight of -0.1 (but note that we had already differentiated them via a weighting scheme in Table 3). All benefit objectives had positive weights. To run the MORTOGA on our SUT, the weight values shown in Table 4 were plugged into the GA's fitness function (Equation 1). Let us recall from the discussions above that, all the cost and benefit factors were normalized into one cost factor (the baseline: Test execution time) and into one unit, staff-hours. We did this normalization to ensure being able to combine all cost and benefit objectives in the fitness function (Equation 1).

As a result of the weighting process, the GA fitness function thus became "dimensionless". In other words, a fitness value calculated by the fitness function represents the cost and value balance of a given solution.

Table 4- Weight values of cost and benefit objectives in the project, used in the MORTOGA's fitness function

\begin{tabular}{|l|l|l|l|}
\hline Cost objectives & Weights & Benefit objectives & Weights \\
\hline Test execution time & -0.1 & Fault severity & 0.2 \\
\hline Third-party cost & -0.1 & Cumulative number of & 0.15 \\
\hline
\end{tabular}


This is a pre-print of the following paper that has been published in the Information and Software Technology (IST) journal: http://dx.doi.org/10.1016/j.infsof.2018.06.007

\begin{tabular}{|l|l|l|l|}
\hline & & detected fault & \\
\hline System setup cost & -0.1 & $\begin{array}{l}\text { Test-suite execution } \\
\text { priority }\end{array}$ & 0.1 \\
\hline Technical resources cost & -0.1 & $\begin{array}{l}\text { Number of detected } \\
\text { fault in the last version }\end{array}$ & 0.05 \\
\hline Verification cost & -0.1 & & \\
\hline
\end{tabular}

\subsection{HOW THE DELPHI METHOD WAS USED FOR DETERMINING WEIGHTS FOR BENEFIT OBJECTIVES}

As discussed above, to quantify benefit objectives and to be able to include those objectives inside the MORTOGA's formula (Equation 1), we used the Delphi method to gather experts' opinions on the importance and thus weights of benefit objectives. We show in Table 5 the steps and activities included in our the Delphi process [76] that we planned and executed. This process was inspired from related works who have used the Delphi method, e.g., [77-81]. There were eight (8) participants in the Delphi estimation team (panel).

Table 5- The wideband Delphi process used in this study

\begin{tabular}{|c|c|}
\hline Phase & Activities \\
\hline Phase 1: Planning & Step 1.1 - Choosing the Delphi estimation team (panel) and a moderator \\
\hline Phase 2: Kickoff meeting & $\begin{array}{l}\text { - Step } 2.1 \text { - The moderator conducted the kickoff meeting, in which the team was presented with the } \\
\text { problem specification and a high-level task list, any assumptions or project constraints. The team } \\
\text { discussed the problem and estimation issues. The moderator guided the entire discussion, } \\
\text { monitored time and after the kickoff meeting, prepared a structured document containing problem } \\
\text { specification, high-level task list, and assumptions that were decided. He then provided the } \\
\text { estimation team with copies of this document for the next step. }\end{array}$ \\
\hline $\begin{array}{l}\text { Phase 3: Independent first } \\
\text { opinions by each team } \\
\text { member }\end{array}$ & $\begin{array}{l}\text { - Step } 3.1 \text { - Each team member was asked to prepare the list of benefit objectives that she/he } \\
\text { considered important, a rank (the most important to least important) and a weight value for each of } \\
\text { those benefit objectives }\end{array}$ \\
\hline $\begin{array}{l}\text { Phase 4: Narrowing down } \\
\text { the benefit objectives and } \\
\text { selecting one single list in a } \\
\text { given rank }\end{array}$ & $\begin{array}{l}\text { - Step 4.1 - At the beginning of the meeting, the moderator collected the list of proposed benefit } \\
\text { objectives, their ranks, and weight values from each of the team members. } \\
\text { - Step 4.2 - Benefit objectives selected by a majority of the panelists were retained } \\
\text { - Step 4.3-After a round of discussions, the panel narrowed down the list of all provided benefit } \\
\text { objectives to the four objectives as shown in Table } 4\end{array}$ \\
\hline $\begin{array}{l}\text { Phase 5: Estimation meeting } \\
\text { to determine weights }\end{array}$ & $\begin{array}{l}\text { - Step } 5.1 \text { - The moderator collected the weight values from all the team members and, for each of } \\
\text { the four objectives, he plotted each member's weight value by a marker on the line of each "round" } \\
\text { (iteration), without disclosing the corresponding team member names. The estimation team then } \\
\text { had an idea of the range of estimates, which initially was quite wide. } \\
\text { - Step } 5.2 \text { - Each team member discussed aloud the rationale behind the weight values benefit } \\
\text { objectives that she/he had chosen, identifying any assumptions made and raising any questions or } \\
\text { issues. } \\
\text { - Step } 5.3 \text { - The team then discussed any doubt/problem they have about the weight values, } \\
\text { assumptions made, and estimation issues. } \\
\text { - Step } 5.5 \text { - Each team member then revisited individually her/his weight values, and makes changes } \\
\text { if necessary. The moderator also made adjustments in the weight values based on the discussions. } \\
\text { - Step 5.6 - The estimation meeting continued by going to be to Step 5.1. The stopping criteria for the } \\
\text { iterations were set as follows: } \\
\text { o Results are converged to an acceptably narrow range. } \\
\text { o All team members are unwilling to change their latest estimates. } \\
\text { o The allotted estimation meeting time was over. } \\
\text { Step } 5.7 \text {-The final weight values were extracted as shown in Table } 4 \text {. }\end{array}$ \\
\hline
\end{tabular}


This is a pre-print of the following paper that has been published in the Information and Software Technology (IST) journal: http://dx.doi.org/10.1016/j.infsof.2018.06.007

The wideband Delphi process for determining weights for benefit objectives had five phases, which started from the planning phase and went on to the last phase (estimation meeting to determine weights).

We executed the Phase 5 with three iterations and, in the last iteration, results converged to an acceptably narrow range, and thus we concluded the meetings. As shown in Table 4, the estimation team (panel), found the "fault severity" objective the most important one and assigned to it a weight value of 0.2 , in the range of $[0,1]$. Cumulative number of detected fault was decided as the second most important benefit objective, with weight $=0.15$. Test-suite execution priority and number of detected fault in the last version were the $3^{\text {rd }}$ and $4^{\text {th }}$ most important objectives.

\subsection{RQ 1: CALIBRATION OF THE MORTOGA}

To run MORTOGA in the project, selection of cost/benefit objectives and data collection for setting the weight values were not enough. As it has been discussed various times in the SBSE community, e.g., $[17,18,61]$, to ensure a GA's best performance, its internal parameters (e.g., population size, crossover and mutation rates) have to be also properly tuned (calibrated) empirically. Many SBSE studies have been conducted in that direction, e.g., [17, 18, 61], and have shown that tuning of a GA has a strong impact on its performance and, thus, finding appropriate parameter values is one of the challenging aspects of applying GAs in SBSE.

The main components of a GA in need of proper tuning are: population size, type of variation operators (mutation and crossover) and rates of applying variation operators [63]. In our study, in order to reach the most sub-optimum solution in acceptable time frames, we calibrated (tuned) the GA parameters including population size, crossover and mutation rates based on our past experience in $[18,61]$, as we discuss next. Calibration of the MORTOGA's parameters was done based on the SUT data for one representative case of regression testing of the SUT, i.e., when the SUT was evolved from version $\# 1$ to version \#2 (see the series of the versions chosen for the empirical study in Section 5.5.1). We calibrated the MORTOGA's parameters before its full "application" on all the SUT versions to ensure the GA would perform well during its full application.

Population Size: Population size means the number of chromosomes (individuals) existing in a GA population. If there are too few chromosomes, the GA will have a few possibilities to perform variations and it will cause the GA to quickly converge to a local minimum [82]. On the other hand, if there are too many chromosomes, the GA may have a performance problem, i.e. its execution time would be too high. The optimal population size for a given GA is the point of "inflection" where the benefit of quick convergence is offset by decreasing performance [82]. In the literature, many studies have suggested adequate population size. De Jong [83] suggests a population size ranging from 50 to 100 chromosomes. Grefenstette et al. [84] recommend a range between 30 and 80, while Schaffer and his colleagues [85] suggest a smaller population size, between 20 and 30 .

In our study, to empirically tune this parameter, similar to our past experience in $[18,61]$, we executed the GA 100 times for each population size value, starting from 5 to 100 with an increasing value of 5 on the case under test (the requirement set in the SUT). In order to assess the GA's performance with each population size setting, we used average values of execution times and fitness values across 100 runs. The dependent variables (metrics) were fitness value and execution times (in seconds). Figure 2 shows the fitness value and execution time curves for the case under test. 
This is a pre-print of the following paper that has been published in the Information and Software Technology (IST) journal: http://dx.doi.org/10.1016/j.infsof.2018.06.007

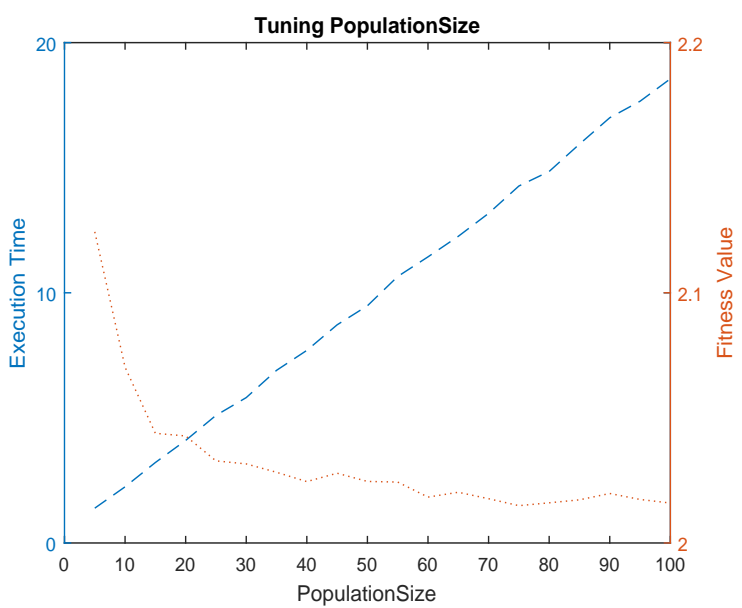

Figure 2- Impact of changes in population size on fitness value and execution time (in seconds)

By looking at these curves, we can see that, in each case, the fitness values do actually converge to a minimum plateau after around the population size of 30. At the same time, as population size increases, execution times increase almost linearly. Thus, a population size of 30-80 could be a good choice for calibration.

Crossover Rate: Crossover rate, a value between [0,1], determines the ratio of how many GA individuals (couples) will be picked for mating and for creating the next set of children for the next generation. Crossover enables a GA to extract the best genes from different individuals and recombine them into potentially "superior" children. Although sources from the literature suggest that the optimal crossover rate is dependent on the nature of structure of the problem at hand, there is a common recommendation of setting the crossover rate to a value between 0.5 and 1 . Here, the value of 1 means full replacement of all the individuals in the current GA generation. Some studies have shown that a crossover rate below 0.5 may decrease the fitness quality [86].

As we did for the case of tuning the population size, we used the same approach for crossover rates as well, i.e., we varied it between 0.5 and 1 with a step value of 0.01 , on the SUT's requirement set. Figure 3 shows the empirical results. By reviewing curves in Figure 3, we see that fitness value remains on a minimum plateau generally between 0.5 and 0.9 crossover rates. We also see that varying the crossover rate have no noticeable effect on execution time, except for values higher than 0.9. Based on this analysis, we decided to use a value between [0.5-0.9] as the suitable calibration for crossover rate. These GA settings were in line with our past experience in [18, 61].

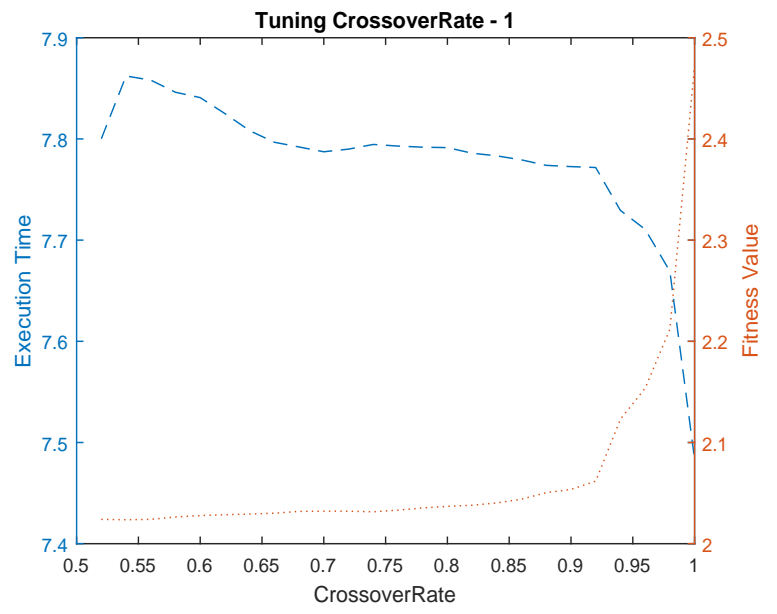


This is a pre-print of the following paper that has been published in the Information and Software Technology (IST) journal: http://dx.doi.org/10.1016/j.infsof.2018.06.007

\section{Figure 3- Impacts of changes in crossover rate on fitness value and execution time}

Mutation Rate: Mutation rate of a GA, a value between [0, 1], determines the ratio of genes in the population that will be flipped (a gene value will be randomly changed). This process adds to the diversity of a population and thereby increases the likelihood that the GA will generate individuals with better fitness values. Generally, many studies have recommended that mutation rate should be a low probability, e.g., [86][83][84][85]. If it is set too high, the search will turn into a primitive random search [86] and GA would stall in converging. In the literature, De Jong [83] suggests a mutation rate of 0.001 . Grefenstette [84] suggests a rate of 0.01, while Schaffer et al. [85] recommended the expression $1.75 / \lambda$ length for the mutation rate where $\lambda$ denotes the population size and length is the length of chromosomes. Mühlenbein [87] suggests a mutation rate defined by $1 /$ length. Based on these recommendations, we decided to empirically evaluate the choices of mutation rates between 1/54 and 27/54 (meaning between 0.01 and 0.5 ) with a step value of $1 / 54$ (54 is the length of chromosome). We used the same approach as the two previous GA parameters, i.e., we executed the GA for 100 times on the requirement set. Figure 4 shows the empirical results of impacts of changes in mutation rate on fitness value and execution time.

By analyzing the trends in Figure 4, we saw that the fitness values reach a minimum plateau after the mutation rate of about 0.07 and then continue with a very small decrease for the rest of the range (0.07-0.5). On the other hand, as mutation rate increases, the execution time also increases quite sharply. Therefore, we decided to tune the GA with a mutation rate between 0.07 and 0.16 .

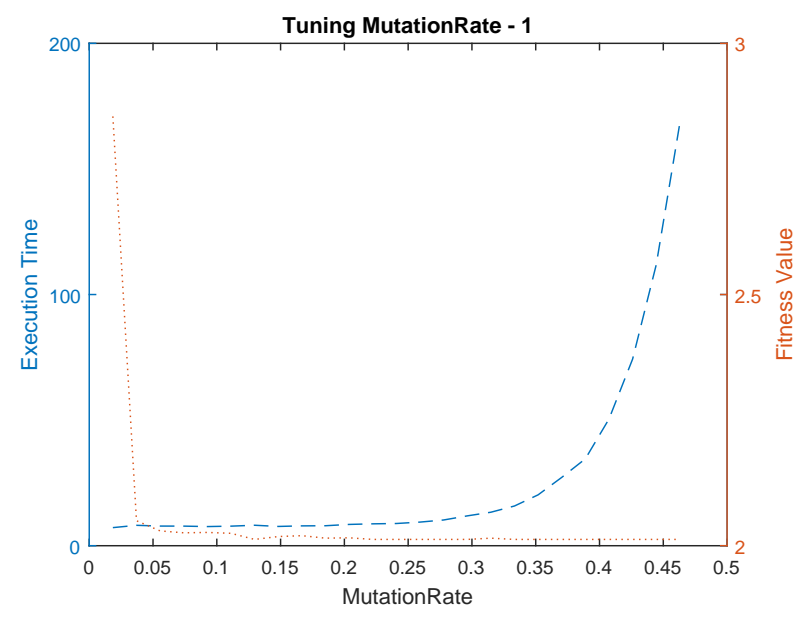

Figure 4- Impacts of changes in mutation rate on fitness value and execution time

Thus, in summary, the good choices for calibration of the three GA parameters are:

- Population size (6 choices): $30,40,50,60,70,80$

- Crossover rate (5 choices): $0.5,0.6,0.7,0.8,0.9$

- Mutation rate (6 choices): $4 / 54=\sim 0.074,5 / 54,6 / 54,7 / 54,8 / 54,9 / 54=\sim 0.16$

Furthermore, to ensure best performance of MORTOGA, we wanted to make sure to choose the most suitable "combination" of best parameter tuning for the three GA parameters. Thus, we considered all the "Cartesian" product of all the above candidate choices which resulted in $180(6 \times 5 \times 6)$ combinations of parameter values. Then, we executed the GA 20 times with each combination of the above parameter values. Thus, we empirically executed in total 180x20=3,600 combinations of parameter values. Note that all executions were controlled automatically (via a script in Matlab [68]) to ensure that we would not spent a large amount of unnecessary manual effort on them. 
This is a pre-print of the following paper that has been published in the Information and Software Technology (IST) journal: http://dx.doi.org/10.1016/j.infsof.2018.06.007

Finally, we picked the parameter combination, which yielded the best fitness values as the best parameter configuration for this empirical study, which was as follows: the value of 40 as population size, 0.7 as crossover rate and 0.12 as mutation rate for the subject case study. In terms of cost (efforts) spent on tuning GWA's parameters, given our experience in this area $[18,61]$, we spent about 30 staff-days for this phase of our work.

\subsection{RQ 2: IMPROVEMENTS IN REGRESSION TESTING PROVIDED BY MORTOGA}

After tuning MORTOGA in RQ1 (previous section), we focused on RQ2 which was the core technical goal of the entire study, via which we wanted to assess the usefulness of MORTOGA in providing improvements in regression testing.

For RQ2, we wanted to measure how much improvement in regression testing the MORTOGA approach provides compared to the manual regression test-selection (as practiced in the industrial context before the project reported in this paper) and an applicable approach from the literature, i.e., the selective requirement coverage-based approach [19]. Our empirical approach to answer RQ2 was to conduct a comparison between the three techniques w.r.t. the following dependent variables: (1) coverage of the affected requirements, and (2) cost/benefit values of regression test-sets of the SUT.

To conduct the empirical study for RQ2, we had to do some set up which we discuss next:

- Choosing the SUT versions for regression testing

- $\quad$ Setting up the control techniques (manual regression testing and selective requirement-based approach)

Afterwards, we report the empirical results in Section 5.5.3 and 5.5.4, which include: coverage of the affected requirements, and cost-benefit analysis of regression testing using either of the three techniques.

\subsubsection{Choosing the SUT versions for regression testing}

For our empirical study of regression testing, we had to select a sequence of SUT versions on which we would apply regression testing using each of the three techniques. For this purpose, we consulted with the test team, and we selected five available builds (versions) of the SUT, for which the manual regression test costs/benefits had been recorded in the project logs in the past and were available for us.

\subsubsection{Setting up the control techniques (manual regression testing and selective requirement-based approach)}

For our empirical study, we also had to properly setup the control techniques which were: manual regression testing approach used in the company in the past, and the selective requirement-based approach [19].

The selective requirement-based approach [19] is a non-linear optimization approach (as is our MORTOGA approach). While most other regression test selection approaches only consider coverage of affected requirements (as reviewed in Section 3) and aims at maximizing it, this approach additionally considers coverage of irrelevant requirement as a second objective to decrease the unnecessary testing effort, by aiming at minimizing it. As defined in [19], the fitness (objective) function of the optimization model used by the approach is as follows:

$$
\mathrm{F}(\mathrm{ts})=\alpha \cdot \mathrm{cc}(\mathrm{ts})+(1-\alpha) \cdot \mathrm{fc}(\mathrm{ts})
$$

Given the weighting factor $\alpha$, for a single test suite $t s$, its fitness function, denoted by $F(t s)$, is defined as the weighted average of its "contribution" criterion $c c(t s)$ and "futile (pointless)" criterion $f c(t s)$ as follows [19]. The contribution criterion of a test suite is the proportion of affected requirements covered by that test suite to the total number of affected requirements. On the other hand, futile criterion is one minus the proportion of irrelevant requirements covered by subject test suite to the total number of irrelevant requirements.

In that approach [19], solving the multi-objective regression test selection problem was defined as a NP-Complete and a heuristic search technique. In the paper which presented the approach [19], it was implemented using a greedy 
This is a pre-print of the following paper that has been published in the Information and Software Technology (IST) journal:

http://dx.doi.org/10.1016/j.infsof.2018.06.007

apporoach, and not a GA. We found that it could indeed be implemented as a GA which would in general be a better solution approach than the greedy approach, due to various reasons, e.g., GAs explore the search space better and with more diversity compared to the greedy apporoach. We used the algorithm and other information as provided in [19] and implemented that approach [19] as a GA in Matlab by using the same chromosome structure as used in MORTOGA (Section 4.2). The source-code of our implementation of that technique [19] in Matlab is available online as open-source [68]. For implementing that approach, we also used the settings (e.g., weighting factor $\alpha$ in the above formula) as suggested in [19].

As for manual regression testing, the team conducted the process as it was established in the past. As discussed in Section 2.2, each time when a software fix was implemented, the customer test team first manually inspected and evaluated the list of affected requirements and the regression test-set proposed by the contractor test team. The customer test team would then either accept the proposed regression test-set or would request adding more test suites to it.

\subsection{Coverage of affected requirements}

We compared the coverage ratios of affected requirements of five different versions of the SUT when using each of the three approaches. Let us recall from earlier sections that affected requirements are those which correspond to the modifications made in the source code across two SUT versions. Figure 5 shows the comparisons. As discussed in Section 2.2 , in the manual (old) approach, the manually-extracted test suites, usually and ironically, did not achieve $100 \%$ coverage of the affected requirements, as we can also see in Figure 5. This is since the contractor test team always tried eagerly to "minimize" the scope of regression test-set, to lower their own test cost, while the customer test team usually tried to "enlarge" the scope of regression test-set by adding extra test suites "to be on the safe side". The end result was usually a test suite which did not achieve full coverage of the affected requirements.

As we can see, only our MORTOGA approach is able to guarantee $100 \%$ coverage of the affected requirements. Since $100 \%$ coverage of affected requirement is a "must" (requirement) for regression testing, the other two approaches are essentially not acceptable w.r.t. this important criterion. Note that the other automated technique (requirement coveragebased approach) [19] also did not provide $100 \%$ coverage of the affected requirements, since, as a search-based approach and according to how it is formulated [19], it "tries" to "maximize" that coverage ratio and, as we can see in Figure 5, it has been able to get close to $100 \%$, but has not reached $100 \%$ precisely.

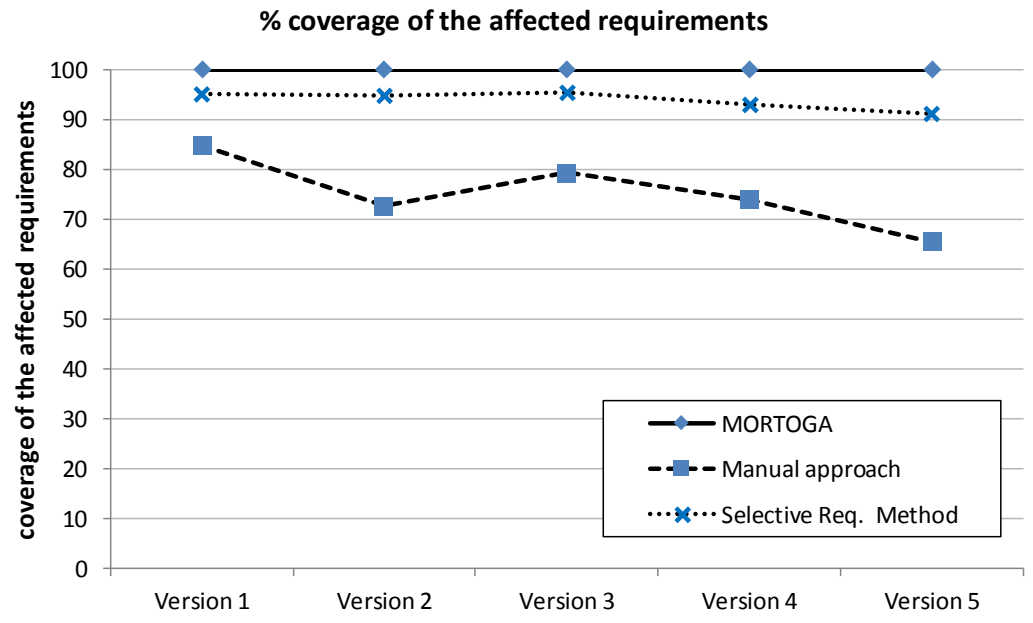

Figure 5- Comparing the three approaches w.r.t. coverage of affected requirements 
This is a pre-print of the following paper that has been published in the Information and Software Technology (IST) journal: http://dx.doi.org/10.1016/j.infsof.2018.06.007

\subsection{Cost and benefit objectives}

Figure 6 shows the comparison of MORTOGA versus the existing manual regression method and the selective requirement-based approach [19] based on the aggregate cost and benefit values. We show in Table 6 all of the cost and benefits measures, for each SUT version, from which we have calculated the aggregate values shown in Figure 6.

The X-axis of the graph in in Figure 6 represents the software versions, while Y-axis shows total cost and benefit values calculated based on the weighting and scaling (normalization) process as explained in Section 5.2, i.e., all five cost objectives are aggregated into one single measure and all four benefit objectives are aggregated into one measure. Note that all the values in Figure 6 and Table 6 are normalized values calculated based on weights assigned to different factors (as discussed in Section 5.2), and thus should not be interpreted with their absolute values.

The comparisons in Figure 6 provide the cost / benefit values of all three methods. To be able to compare the three techniques for benefit versus cost measures over all the five versions together, we show in the last column of Table 6 the sum of all values in each row. For example, for the manual regression test selection approach, totals of cost and benefit values are 18.32 and 27.21. To compare the three techniques, we computed the ratio of benefit/cost total values, which are 1.48 (=27.21/18.32), 3.79 (=33.90/8.94), and $1.97(=29.18 / 14.79)$, respectively, for the three techniques listed in Table 6. As these ratios show and as we can visually see in Figure 6, MORTOGA incurs lower cost than the other two methods when aggregating all of the five versions, while it also provides higher benefits in three of the five versions (versions $1 \ldots 5$ ).

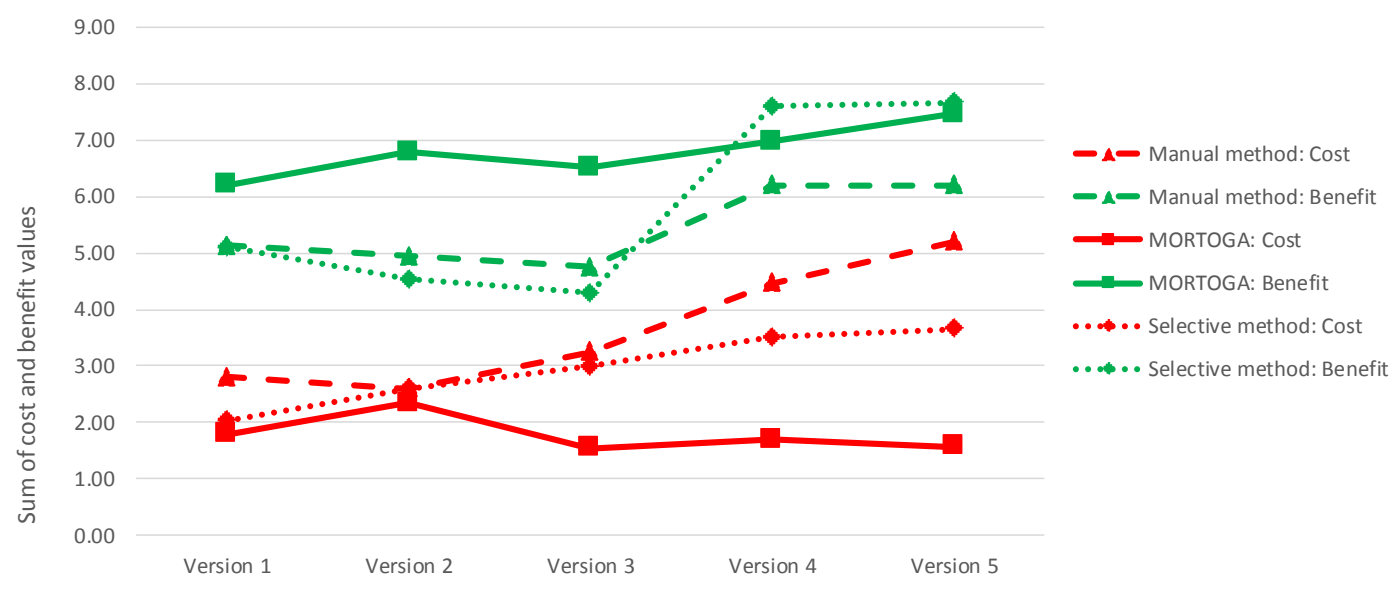

Figure 6 - Comparing the three approaches w.r.t. aggregate cost and benefit values

Thanks to the search-based approach and optimization engine built in MORTOGA, it efficiently searches among the large number of test-case combinations and generates as output a set of test suites which is the most efficient regression test suite, when executed on the SUT, by considering the set of cost and benefit objectives.

An important point that should be discussed is about the following fault-related metrics shown in Table 6: number of detected faults (cumulative across all SUT versions so far), and number of detected faults (in the last SUT version only). As discussed above, all these measures are normalized values calculated based on weights assigned to different cost/benefit factors (as discussed in Section 5.2), and thus are not necessarily integer numbers. Of course, when one deals with faults, the measures will be absolute (integer) numbers. However, since weights are involved in calculating these normalized values, the outcomes are real numbers (as shown in Table 6). Also, we should note that the fault-related metrics shown in Table 6 are only from the application of the MORTOGA and the selective requirement coverage method to derive the best regression test suites based on historical fault data; and the fault-related metrics are not for the actual number of faults found by executing the best regression test suites generated by each of those techniques. As we discuss in Section 5.6, although the MORTOGA approach is currently in active use in the company, not all the generated 
This is a pre-print of the following paper that has been published in the Information and Software Technology (IST) journal:

http://dx.doi.org/10.1016/j.infsof.2018.06.007

regression test suites detect real faults and thus we could not use those test-execution data for estimating fault detection effectiveness. Instead, we would have liked to apply fault injection (mutation testing) on the SUT to assess the effectiveness of the regression test suites generated by these two techniques, but as discussed in Section 2.2, due to the classified nature of the SUT, neither we nor the contractor test team had access to the SUT's source code, thus mutation testing was not possible.

Instead, we found another way for estimating fault detection effectiveness based on the available data. We have the number of test suites generated by the approach and the number of potentially detected faults (cumulative), form historical fault data (both shown in Table 6). We thus divided the latter by the former, and estimated the "potential" fault detection ability of each output test suite, for each SUT version. For example, for version 2, MORTOGA selected 10 of the all 54 system test suites for regression testing and they have the potential to detect a normalized value of 1.71 faults, thus the fault detection ability is 0.17 . When we added up all the fault detection effectiveness values for all five SUT versions for each technique, the total values are 0.74, 1.00, 0.79, respectively, for the three approaches. Again, MORTOGA has the best performance w.r.t. this metric.

From another perspective, another point that got our attention was the "fluctuations" in cost and benefit measures across the three methods among the five versions of the SUT in Figure 6. We got curious about that issue and assessed the associated root cause(s). We found that those fluctuations are due to a number of factors, e.g., (1) the number of affected requirements in each version, as there were 310, 314, 212, 169 and 113 affected requirements, respectively, for each of the five versions; and (2) the correspondence (traceability) of test steps in different test suites to those affected requirements.

We also observe in Figure 6 that the other automated technique (selective requirement coverage-based approach [19]) has provided a greater "benefit" value in one of the five cases (version 4 of the SUT). However, the major issue with that other technique is that it does not "guarantee" reaching 100\% coverage of the affected requirements, as we empirically observed in Section 5.5.2 (and in Figure 5). Thus, although it provides better "benefit" for version 4 of the SUT, it fails to be an acceptable solution approach.

Table 6-Details of cost and benefits measures for the three approaches for the five versions of the SUT

\begin{tabular}{|c|c|c|c|c|c|c|c|}
\hline \multirow[t]{2}{*}{ Methods } & \multirow[t]{2}{*}{ Cost/benefit factors } & \multicolumn{5}{|c|}{ Versions of the SUT } & \multirow[t]{2}{*}{ Sum } \\
\hline & & $\# 1$ & $\# 2$ & $\# 3$ & $\# 4$ & $\# 5$ & \\
\hline \multirow{13}{*}{$\begin{array}{l}\text { Manual } \\
\text { regression } \\
\text { test } \\
\text { selection } \\
\text { approach }\end{array}$} & Execution time (hour) & 0.86 & 0.68 & 0.93 & 0.93 & 1.31 & 4.71 \\
\hline & Third -party costs & 0.21 & 0.55 & 0.00 & 0.92 & 1.25 & 2.93 \\
\hline & Setup costs & 0.61 & 0.40 & 0.75 & 0.80 & 1.31 & 3.87 \\
\hline & Verification (oracle) costs & 0.02 & 0.41 & 0.76 & 0.81 & 0.00 & 2.01 \\
\hline & Technical resources costs (test exec environment) & 1.10 & 0.56 & 0.81 & 1.00 & 1.32 & 4.81 \\
\hline & Total costs & 2.81 & 2.60 & 3.25 & 4.46 & 5.19 & 18.32 \\
\hline & \# of detected faults (cumulative) -normalized & 1.48 & 1.32 & 1.23 & 1.64 & 1.57 & 7.75 \\
\hline & \# of detected faults (last version)-normalized & 0.38 & 0.66 & 0.81 & 1.10 & 1.29 & 4.23 \\
\hline & Faults severity & 1.54 & 1.45 & 1.34 & 1.80 & 1.63 & 7.99 \\
\hline & Stakeholder priority $(1,2$ or 3$)$ & 1.73 & 1.50 & 1.38 & 1.66 & 1.72 & 7.24 \\
\hline & Total benefits & 5.13 & 4.94 & 4.75 & 6.20 & 6.20 & 27.21 \\
\hline & Number of test suites generated by the approach & 20 & 13 & 9 & 10 & 6 & 58 \\
\hline & Potential fault detection ability of the test suites & 0.07 & 0.10 & 0.14 & 0.16 & 0.26 & 0.74 \\
\hline \multirow{6}{*}{ MORTOGA } & Execution time (hour) & 0.54 & 0.54 & 0.44 & 0.40 & 0.33 & 2.26 \\
\hline & Third -party costs & 0.32 & 0.44 & 0.51 & 0.33 & 0.30 & 1.90 \\
\hline & Setup costs & 0.27 & 0.42 & 0.00 & 0.26 & 0.30 & 1.24 \\
\hline & Verification (oracle) costs & 0.26 & 0.42 & 0.27 & 0.27 & 0.30 & 1.52 \\
\hline & Technical resources costs (test exec environment) & 0.40 & 0.53 & 0.32 & 0.44 & 0.34 & 2.02 \\
\hline & Total costs & 1.79 & 2.34 & 1.54 & 1.70 & 1.57 & 8.94 \\
\hline
\end{tabular}


This is a pre-print of the following paper that has been published in the Information and Software Technology (IST) journal: http://dx.doi.org/10.1016/j.infsof.2018.06.007

\begin{tabular}{|c|c|c|c|c|c|c|c|}
\hline & \# of detected faults (cumulative) -normalized & 1.67 & 1.71 & 1.65 & 1.79 & 1.86 & 8.69 \\
\hline & \# of detected faults (last version) -normalized & 0.85 & 1.22 & 1.30 & 1.34 & 1.58 & 6.30 \\
\hline & Faults severity & 1.91 & 1.81 & 1.75 & 1.89 & 1.94 & 9.63 \\
\hline & Stakeholder priority $(1,2$ or 3$)$ & 1.77 & 2.04 & 1.80 & 1.95 & 2.07 & 8.69 \\
\hline & Total benefits & 6.20 & 6.77 & 6.50 & 6.97 & 7.46 & 33.90 \\
\hline & Number of test suites generated by the approach & 13 & 10 & 7 & 9 & 7 & 46 \\
\hline & Potential fault detection ability (Ratio) & 0.13 & 0.17 & 0.24 & 0.20 & 0.27 & 1.00 \\
\hline \multirow{13}{*}{$\begin{array}{l}\text { Selective } \\
\text { requirement } \\
\text { coverage } \\
\text { method }\end{array}$} & Execution time (hour) & 0.63 & 0.63 & 0.73 & 0.76 & 0.97 & 3.73 \\
\hline & Third -party costs & 0.31 & 0.49 & 1.09 & 0.69 & 0.85 & 3.44 \\
\hline & Setup costs & 0.36 & 0.47 & 0.00 & 0.62 & 0.91 & 2.36 \\
\hline & Verification (oracle) costs & 0.31 & 0.44 & 0.56 & 0.63 & 0.00 & 1.94 \\
\hline & Technical resources costs (test exec environment) & 0.41 & 0.56 & 0.61 & 0.80 & 0.93 & 3.32 \\
\hline & Total costs & 2.03 & 2.60 & 2.99 & 3.51 & 3.66 & 14.79 \\
\hline & \# of detected faults (cumulative) -normalized & 1.37 & 1.19 & 1.10 & 1.95 & 1.95 & 7.56 \\
\hline & \# of detected faults (last version) -normalized & 0.76 & 0.70 & 0.75 & 1.50 & 1.64 & 5.35 \\
\hline & Faults severity & 1.48 & 1.30 & 1.19 & 2.04 & 2.01 & 8.03 \\
\hline & Stakeholder priority $(1,2$ or 3$)$ & 1.49 & 1.35 & 1.25 & 2.10 & 2.05 & 8.25 \\
\hline & Total benefits & 5.10 & 4.54 & 4.30 & 7.59 & 7.66 & 29.18 \\
\hline & Number of test suites generated by the approach & 12 & 10 & 8 & 8 & 11 & 49 \\
\hline & Potential fault detection ability of the test suites & 0.11 & 0.12 & 0.14 & 0.24 & 0.18 & 0.79 \\
\hline
\end{tabular}

\subsection{BENEFITS OF THE RESULTS AND THE MORTOGA APPROACH IN THE INDUSTRIAL CONTEXT}

Since the MORTOGA approach has development and calibration efforts associated with it, we decided to objectively and critically evaluate its benefit for the test team. One of the authors had done a similar analysis in a previous work [88], in which he and his colleagues quantitatively estimated the time saving (cost-benefit) of a novel automated testing platform for an industrial partner.

To objectively and critically justify the development and calibration efforts (costs) of MORTOGA in the industrial context, we looked at the costs values of the three approaches in Table 6, and aimed at estimating how much cost saving MORTOGA would provide. Let us recall from Section 2.1 that we had an estimate about the total manual execution time of the entire test suites (187+ staff-days). Thus, if we could estimate how much reduction in total manual execution time, the output test suite by MORTOGA would provide, we would be able to justify using MORTOGA.

As discussed in Section 5.5.2. since 100\% coverage of affected requirement is a "must" (requirement) for regression testing, the other automated technique [19] was essentially not acceptable w.r.t. this important criterion and we thus only compared the manual (old) approach and MORTOGA's costs (including its development and calibration efforts). Over the five versions, test execution time of the test suites selected by the manual (old) approach had a cost of 4.71 in the unit of analysis (recall that values are normalized), while MORTOGA had a cost of only 2.26. This mean a cost saving of 52.1\% of the total manual execution time of the entire test suites. Thus, we expected that using MORTOGA would save about 97 staff-days $(187 \times 52.1 \%)$ in that manual execution effort. Further note that such a saving is just for one single application of MORTOGA on the pilot five-version series of the SUT, and we should note that regression testing of the SUT have been continuously conducted since several years ago and will go on for several years to come (the project has no "end date" yet as of this writing). According to our time logs, development and calibration of MORTOGA took about 200 staff-days in total. Thus we expect that using MORTOGA in two cases such as the one we reported in this paper would "pay off" its development and calibration costs.

From another perspective, as we discussed in the related work (Section 3), most of the regression test-selection techniques proposed in literature are source-code based and they are not applicable for the cases when source code is not available (such as our case). Most of the regression test-selection techniques presented in literature are structured based on a single 
This is a pre-print of the following paper that has been published in the Information and Software Technology (IST) journal: http://dx.doi.org/10.1016/j.infsof.2018.06.007

objective. However, in real-world regression testing scenarios, there are many different criteria that may have impact on effectiveness of a test suite like execution time, coverage, user priority, fault history etc. The use of a single criterion does not answer regression test selection requirements in practice and it severely limits its fault detection ability.

The results of this study show that MORTOGA provides a suitable solution option for both issues mentioned above. With $100 \%$ coverage of affected requirements, it provides a high-level (requirement coverage based) solution option especially for the large-scale complex systems. In terms of optimization, MORTOGA effectively selects the optimum solution based on real-world cost and benefit objectives. This aspect of the approach provides more realistic solutions than single objective approaches. Since the proposed approach (MORTOGA) has been beneficial in the industrial context in saving the costs of regression testing (as shown above), it is currently in active use in the company and project under study. Since the manual regression test-selection approach was in use before we had developed MORTOGA, we used the empirical results as reported in this paper, as motivations to communicate with the test team's management. Since the entire study was closely conducted with participation of the test team and its management, "deploying" the MORTOGA approach was straightforward and, with some time spent on briefing test team, the entire team "embraced" this new and better approach and the method has been in active use, as of this writing.

\subsection{LIMITATIONS AND THREATS TO VALIDITY}

This empirical study was an action-research [13, 14] project in which we developed a solution method based on optimization search. The purpose of the study was to apply a tailored version of a MORTO approach [10] to an industrial project and assess the effectiveness of proposed methodology by comparing the existing manual regression testing approach with the proposed solution.

The validity of a study denotes the trustworthiness of the results, and to what extent the results are true and not biased by the researchers' subjective point of view [89]. We discuss next the potential threats to the validity of our study and the steps that we have taken to minimize or mitigate them. The threats are discussed in the context of the four types of threats to validity based on a standard checklist for validity threats presented in [90]: internal validity, construct validity, conclusion validity and external validity.

Internal validity: Internal validity in this study deals with designing the experiment correctly (i.e., without having confounding factors, bias, etc.) such that any observed effect in the dependent variables can really be attributed to the change of independent variables (in our case, the change of the regression test selection method). Internal validity of the study was ensured since all approaches were developed and applied systematically. A potential threat to internal validity in this experiment could be the poor setting of GA parameters. That threat was minimized by taking a systematic approach to systematically tune the GA parameters for the proposed solution, as discussed in Section 5.3. Another potential threat to internal validity which is also a treat to conclusion validity could the relative small scale of the case under study. We believe the SUT was a mid-size software since it includes 935 system-level requirements. To verify those system-level requirements, 54 different test suites were developed, each containing between 182 to 3,588 test steps. Thus, the context is a typical (representative) industrial context, as per our experience in testing various types of systems in the past, e.g. [11].

Construct validity: Construct validity is concerned with the extent to which the object of study truly represents theory behind the study [89]. We are not aware of any construct validity in this study.

Conclusion validity: Conclusion validity is concerned with the correct application of statistical procedures (which in our case does not apply) and the correct interpretation of the results of the experiment. For this empirical study, one possible threat is about running the GA which is a stochastic algorithm and a single run of this algorithm may not provide a stable result. In order to mitigate that threat, we used our past experience in GA parameter tuning [18, 61], and executed all the GA-related experiments for a large number of times (at least, 100) and we picked the best outcomes. 
This is a pre-print of the following paper that has been published in the Information and Software Technology (IST) journal: http://dx.doi.org/10.1016/j.infsof.2018.06.007

External validity: External validity is concerned with the extent to which the results of this study can be generalized. Threats to external validity are conditions that could limit our ability to generalize the results of our experiment to industrial practice. As mentioned before, this study was an action-research project. It presented a solution for a specific industrial problem with specific parameters and settings. However, our solution provides a general and flexible structure which is applicable to a typical regression test-selection problem, in black-box approach (based on requirements). For example, as discussed in Section 4.2, we developed MORTOGA in a flexible / extensible manner in terms of the cost/benefit objectives, i.e., various sets of cost and benefit objectives can be plugged into the fitness function, depending on the system and project under test.

\section{CONCLUSIONS AND FUTURE WORK}

Based on the principles of "action-research" [13, 14], we presented in this paper a GA-based approach (called MORTOGA) and an empirical study on regression test-selection problem of an industrial project with several cost and benefit objectives. Two research questions were raised and answered in scope of the empirical study: (RQ1) How can the GA be best calibrated to yield the best results for the context at hand?; and (RQ2) How much improvements does the approach provide compared to the previous existing test selection approach?

We formulated and solved the problem using a custom-built genetic algorithm (GA). The empirical results demonstrated that this approach yields a more efficient regression test-set in terms of cost/benefit and more effective in terms of coverage of affected requirements compared to the old (manual) test-selection approach, and another approach from the literature, i.e., the selective requirement coverage-based approach [19]. We developed MORTOGA based on a set of five cost objectives and four benefit objectives for regression testing while providing full coverage of affected requirements.

Since our proposed approach has been beneficial and has made a positive difference in the company practices in terms of regression testing, it is currently in active use in the industrial context. With this approach, regression selection process in the project is not ad-hoc anymore. Furthermore, we were able to eliminate the subjectivity of regression testing and its dependency on expert opinions. Thanks to the new approach, regression test-sets are now determined based on requirement coverage, and cost and benefits of test suites in a systematic approach.

Our future work directions include the followings: (1) enhancement of MORTOGA by covering other cost and benefit parameters; (2) applying MORTOGA in other contexts and regression test-selection problems and to further assess its generalizability; and (3) further assessment of the fault detection effectiveness of the three approaches by conducting mutation testing. Due to confidentiality, logistical and costs reasons, we were so far unable to inject artifacts defects (mutants) in the real SUT in the field, and measure fault detection rates (mutation scores).

\section{Acknowledgements:}

The authors would like to thank Dietmar Pfahl for a detailed review on an early draft of this paper.

\section{REFERENCES}

[1] Tricentis Inc., "'Software fail watch" report, 5th edition," https://www.tricentis.com/software-fail-watch/, 2017 , Last accessed: Jan. 2018.

[2] Research Triangle Institute, "The economic impacts of inadequate infrastructure for software testing," NIST Planning Report 02-3, National Institute of Standards and Technology, 2002.

[3] E. Torres, "Inadequate Software Testing Can Be Disastrous," IEEE Potentials, vol. 37, no. 1, pp. 9-47, 2018.

[4] S. Yoo and M. Harman, "Regression Testing Minimization, Selection and Prioritization: A Survey," Softw. Test. Verif. Reliab., vol. 22, pp. 67-120, 2012.

[5] M. Vierhauser, R. Rabiser, P. Gr, \#252, and nbacher, "A case study on testing, commissioning, and operation of very-large-scale software systems," presented at the Companion Proceedings of International Conference on Software Engineering, Hyderabad, India, 2014.

[6] R. Carlson, H. Do, and A. Denton, "A clustering approach to improving test case prioritization: An industrial case study," presented at the Proceedings of IEEE International Conference on Software Maintenance, 2011.

[7] C. Henard, M. Papadakis, M. Harman, Y. Jia, and Y. L. Traon, "Comparing White-Box and Black-Box Test Prioritization," in IEEE/ACM International Conference on Software Engineering, 2016, pp. 523-534. 
This is a pre-print of the following paper that has been published in the Information and Software Technology (IST) journal: http://dx.doi.org/10.1016/j.infsof.2018.06.007

[8] R. K. Saha, L. Zhang, S. Khurshid, and D. E. Perry, "An Information Retrieval Approach for Regression Test Prioritization Based on Program Changes," in IEEE/ACM International Conference on Software Engineering, 2015, vol. 1, pp. 268-279.

[9] E. Engström, P. Runeson, and M. Skoglund, "A systematic review on regression test selection techniques," Information and Software Technology, vol. 52, pp. 14-30, 2010.

[10] M. Harman, "Making the case for MORTO: Multi objective regression test optimization," Proceedings International Conference on Software Testing, Verification, and Validation Workshops, pp. 111-114, 2011.

[11] V. Garousi, M. M. Eskandar, and K. Herkiloğlu, "Industry-academia collaborations in software testing: experience and success stories from Canada and Turkey," Software Quality Journal, special issue on Industry Academia Collaborations in Software Testing, pp. 1-53, 2016.

[12] V. Garousi, K. Petersen, and B. Özkan, "Challenges and best practices in industry-academia collaborations in software engineering: a systematic literature review," Information and Software Technology, vol. 79, pp. 106-127, 2016.

[13] P. S. M. d. Santos and G. H. Travassos, "Action research use in software engineering: An initial survey," in Proceedings of International Symposium on Empirical Software Engineering and Measurement, 2009, pp. 414-417, 1671296.

[14] D. E. Avison, F. Lau, M. D. Myers, and P. A. Nielsen, "Action research," Communications of the ACM, vol. 42, no. 1, pp. 94-97, 1999.

[15] S. N. Sivanandam and S. N. Deepa, Introduction to Genetic Algorithms. Springer, 2007.

[16] T. Gorschek, P. Garre, S. Larsson, and C. Wohlin, "A Model for Technology Transfer in Practice," IEEE Software, vol. 23, no. 6, pp. 88-95, 2006.

[17] A. Arcuri and G. Fraser, "On parameter tuning in search based software engineering," in Proceedings of international conference on Search-based software engineering, 2011, pp. 33-47, 2042252.

[18] V. Garousi, "Empirical analysis of a genetic algorithm-based stress test technique," presented at the Proceedings of annual conference on Genetic and evolutionary computation, 2008.

[19] Q. Gu, B. Tang, and D. Chen, "Optimal Regression Testing based on Selective Coverage of Test Requirements," in International Symposium on Parallel and Distributed Processing with Applications, 2010, pp. 419-426.

[20] R. Özkan, "Multi-objective regression test selection in practice: an empirical study in the defense software industry," MSc thesis, Middle East Technical University, http://etd.lib.metu.edu.tr/upload/12620692/index.pdf, 2017, Last accessed: Jan. 2018.

[21] V. Garousi and M. V. Mäntylä, "When and what to automate in software testing? A multivocal literature review," Information and Software Technology, vol. 76, pp. 92-117, 2016.

[22] B. Xiaoying, W. T. Tsai, R. Paul, S. Techeng, and L. Bing, "Distributed end-to-end testing management," in Proceedings IEEE International Enterprise Distributed Object Computing Conference, 2001, pp. 140-151.

[23] J.-M. K. J.-M. Kim and a. Porter, "A history-based test prioritization technique for regression testing in resource constrained environments," Proceedings of the 24th International Conference on Software Engineering. ICSE 2002, pp. 119-129, 2002.

[24] K. Deb, Multi-Objective Optimization Using Evolutionary Algorithms. John Wiley \& Sons, 2001.

[25] S. Biswas, R. Mall, M. Satpathy, and S. Sukumaran, "Regression test selection techniques: A survey," Informatica, 2011.

[26] Y. Singh, "Systematic Literature Review on Regression Test Prioritization Techniques," Informatica vol. 36, pp. 379-408, 2012.

[27] M. Khatibsyarbini, M. A. Isa, D. N. A. Jawawi, and R. Tumeng, "Test case prioritization approaches in regression testing: A systematic literature review," Information and Software Technology, vol. 93, pp. 74-93, 2018.

[28] C. Catal and D. Mishra, "Test case prioritization: a systematic mapping study," Software Quality Journal, vol. 21, no. 3, pp. 445-478, 2013.

[29] V. Garousi and M. V. Mäntylä, "A systematic literature review of literature reviews in software testing," Information and Software Technology, vol. 80, pp. 195-216, 2016.

[30] B. Qu, C. Nie, B. Xu, and X. Zhang, "Test Case Prioritization for Black Box Testing," in Proceedings of International Computer Software and Applications Conference, 2007, pp. 465-474.

[31] R. Gupta, M. J. Harrold, and M. L. Soffa, "An approach to regression testing using slicing," Proceerdings of Int. Conference on Software Maintenance, pp. 299-308, 1992.

[32] G. Rothermel, "Efficient, Effective Regression Testing Using Safe Test Selection Techniques," ed. Doctoral Dissertation, Clemson University, 1996.

[33] G. Rothermel and M. J. Harrold, "A safe, efficient regression test selection technique," ACM Transactions on Software Engineering and Methodology, vol. 6, pp. 173-210, 1997.

[34] S. Bates and S. Horwitz, "Incremental program testing using program dependence graphs," Proceedings of symposium on Principles of programming languages, vol. 5, pp. 384-396, 1993.

[35] W. E. Wong, J. R. Horgan, S. London, H. Agrawal, and S. Street, "A Study of Effective Regression Testing in Practice," in Proceedings of the International Symposium on Software Reliability Engineering, 1997, pp. 264-274.

[36] J. a. Jones and M. J. Harrold, "Test-suite reduction and prioritization for modified condition/decision coverage," in IEEE International Conference on Software Maintenance, 2001, pp. 92-103.

[37] Y. Chen, R. L. Probert, and H. Ural, "Model-based Regression Test Suite Generation Using Dependence Analysis," in Proceedings of the international workshop on Advances in model-based testing, 2007, pp. 54-62.

[38] M. Akhin and V. Itsykson, "A regression test selection technique based on incremental dynamic analysis," Software Engineering Conference in Russia, pp. 19-24, 2009.

[39] V. Garousi, E. G. Ergezer, and K. Herkiloğlu, "Usage, usefulness and quality of defect reports: an industrial case study in the defence domain," in International Conference on Evaluation and Assessment in Software Engineering (EASE), 2016, pp. 277-282. 
This is a pre-print of the following paper that has been published in the Information and Software Technology (IST) journal: http://dx.doi.org/10.1016/j.infsof.2018.06.007

[40] M. E. Coşkun, M. M. Ceylan, K. Yiğitözu, and V. Garousi, "A tool for automated inspection of software design documents and its empirical evaluation in an aviation industry setting," in Proceedings of the International Workshop on Testing: Academic and Industrial Conference - Practice and Research Techniques (TAIC PART), 2016, pp. 118-128.

[41] V. Garousi et al., "Experience in automated testing of simulation software in the aviation industry," IEEE Software, In Press, 2017.

[42] B. Chase, "Portable Automated Test Station: Using Engineering-Design Partnerships to Replace Obsolete Test Systems," CrossTalk, The Journal of Defense Software Engineering, pp. 4-8, 2015.

[43] C. Hagen, S. Hurt, and A. Williams, "Metrics That Matter in Software Integration Testing Labs," CrossTalk, The Journal of Defense Software Engineering, pp. 24-28, 2015.

[44] J. Park, H. Ryu, H.-J. Choi, and D.-K. Ryu, "A survey on software test maturity in Korean defense industry," presented at the Proceedings of the India software engineering conference, Hyderabad, India, 2008.

[45] A. Shumskas, "Software test and analysis: Department of Defense policy directions," in Proceedings of Annual International Computer Software E Applications Conference, 1989, p. 526.

[46] B. Gauf, "Defense Needs Better Ways to Test Software," National Defense Magazine, http://www.nationaldefensemagazine.org/articles/2013/10/1/2013october-defense-needs-better-ways-to-test-software, 2013, Last accessed: Jan. 2018.

[47] B. Robinson, "Trustworthy battlefield software depends on better testing," Defense Systems Magazine, https://defensesystems.com/articles/2010/06/07/defense-it-software-testing.aspx, 2010, Last accessed: Jan. 2018.

[48] US Department of Defense, "Software Testing During Post-Development Support of Weapons Systems," Report No. 94-175, 1994.

[49] M. Reginald N, C. Youngblut, and D. A. Wheeler, "Software Testing Initiative for Strategic Defense Systems," Tehcnical report P-2701, Institute for Defense Analyses, 1992.

[50] S. Biffl, A. Aurum, B. Boehm, H. Erdogmus, and P. Grünbacher, Value-Based Software Engineering. Springer, 2010.

[51] B. W. Boehm, Software Engineering Economics. Prentice Hall, 1981.

[52] S. Yoo and M. Harman, "Pareto efficient multi-objective test case selection," in Proceedings of international symposium on Software testing and analysis, 2007, p. 140 .

[53] A. D. Lucia, M. D. Penta, R. Oliveto, and A. Panichella, "On the role of diversity measures for multi-objective test-case selection," in International Workshop on Automation of Software Test, 2012, pp. 145-151.

[54] L. S. d. Souza, R. B. C. Prudencio, and F. d. A. Barros, "Multi-Objective Test Case Selection: A study of the influence of the Catfish effect on PSO based strategies," in Workshop de Testes e Tolerância a Falhas - WTF, 2014

[55] M. G. Epitropakis, S. Yoo, M. Harman, and E. K. Burke, "Empirical evaluation of pareto efficient multi-objective regression test case prioritisation," in Proceedings of International Symposium on Software Testing and Analysis, 2015, pp. 234-245, 2771788.

[56] D. Mondal, H. Hemmati, and S. Durocher, "Exploring Test Suite Diversification and Code Coverage in Multi-Objective Test Case Selection," in International Conference on Software Testing, Verification and Validation, 2015, pp. 1-10.

[57] "Multi-objective optimization," Wikipedia, 2016. [Online]. Available.

[58] "Heuristic (computer science)," Wikipedia, 2016. [Online]. Available.

[59] M. Harman, P. McMinn, J. T. de Souza, and S. Yoo, "Search Based Software Engineering: Techniques, Taxonomy, Tutorial," in Empirical Software Engineering and Verification: International Summer Schools, B. Meyer and M. Nordio, Eds., 2012, pp. 1-59.

[60] V. Garousi, "Traffic-aware Stress Testing of Distributed Real-Time Systems based on UML Models using Genetic Algorithms," Journal of Systems and Software, vol. 81, no. 2, pp. 161-185, 2006.

[61] M. R. Karim, G. Ruhe, M. M. Rahman, V. Garousi, and T. Zimmermann, "An empirical investigation of single-objective and multi-objective evolutionary algorithms for developer's assignment to bugs," Journal of Software: Evolution and Process, vol. 28, no. 12, pp. 1025-1060, 2016.

[62] S. Winkler and J. Pilgrim, "A survey of traceability in requirements engineering and model-driven development," Software and System Modeling, vol. 9, no. 4, pp. 529-565, 2010.

[63] A. E. Eiben and J. E. Smith, "What is an Evolutionary Algorithm?," Introduction to Evolutionary Computing, pp. 15-35, 2003.

[64] K. Miettinen and M. M. Mäkelä, "On scalarizing functions in multiobjective optimization," OR Spectrum, vol. 24, no. 2, pp. 193-213, 2002.

[65] S. Yoo and M. Harman, "Pareto efficient multi-objective test case selection," Proceedings of the 2007 international symposium on Software testing and analysis - ISSTA '07, p. 140, 2007.

[66] G. Eichfelder, Adaptive Scalarization Methods in Multiobjective Optimization. Springer, 2008.

[67] H. Ishibuchi, T. Doi, and Y. Nojima, "Incorporation of Scalarizing Fitness Functions into Evolutionary Multiobjective Optimization Algorithms," in International Conference on Parallel Problem Solving from Nature, 2006, pp. 493-502.

[68] R. Özkan, V. Garousi, and A. Betin-Can, "GA tool source-code in Matlab for multi-objective regression testing," http://doi.org/10.5281/zenodo.1149058, 2017.

[69] J. Zander, I. Schieferdecker, and P. J. Mosterman, Model-Based Testing for Embedded Systems. CRC Press, 2011.

[70] V. Garousi, M. Felderer, Ç. M. Karapıçak, and U. Yılmaz, "What we know about testing embedded software," IEEE Software, In press, 2018.

[71] M. Jørgensen, "A review of studies on expert estimation of software development effort," Journal of Systems and Software, vol. 70, no. 1, pp. 3760, 2004.

[72] A. Idri, F. a. Amazal, and A. Abran, "Analogy-based software development effort estimation: A systematic mapping and review," Information and Software Technology, vol. 58, pp. 206-230, 2015.

[73] E. Aranha and P. Borba, "An Estimation Model for Test Execution Effort," in International Symposium on Empirical Software Engineering and Measurement, 2007, pp. 107-116. 
This is a pre-print of the following paper that has been published in the Information and Software Technology (IST) journal: http://dx.doi.org/10.1016/j.infsof.2018.06.007

[74] S. Elbaum, A. G. Malishevsky, and G. Rothermel, "Prioritizing test cases for regression testing," SIGSOFT Software Engineering Notes, vol. 25, no. 5 , pp. 102-112, 2000.

[75] T. Zimmermann, N. Nagappan, and A. Zeller, "Predicting Bugs from History," in Software Evolution: Springer Berlin Heidelberg, 2008, pp. 6988.

[76] M. Adler and E. Ziglio, Gazing Into the Oracle: The Delphi Method and Its Application to Social Policy and Public Health. Jessica Kingsley Publishers, 1996.

[77] T. J. Gandomani, K. T. Wei, and A. K. Binhamid, "A case study research on software cost estimation using experts' estimates, Wideband Delphi, and Planning Poker technique," International Journal of Software Engineering and its Applications, Article vol. 8, no. 11, pp. 173-182, 2014.

[78] A. Rai, G. P. Gupta, and P. Kumar, "Estimation of software development efforts using improved delphi technique: A novel approach," International Journal of Applied Engineering Research, Article vol. 12, no. 12, pp. 3228-3236, 2017.

[79] S. Piroozfar, R. Barzi, and M. A. A. Kazemi, "Identify software quality indicators based on Fuzzy Delphi method," in Proceedings of International Conference on Computers and Industrial Engineering, 2015.

[80] T. Philip, G. Schwabe, and E. Wende, "Identifying early warning signs of failures in offshore software development projects - a Delphi survey," in Proc. of Americas Conference on Information Systems, 2010, pp. 3925-3934.

[81] R. Schmidt, K. Lyytinen, M. Keil, and P. Cule, "Identifying Software Project Risks: An International Delphi Study," Journal of Management Information Systems, vol. 17, no. 4, pp. 5-36, 2001.

[82] S. Gotshall and B. Rylander, "Optimal population size and the genetic algorithm," Proceedings On Genetic And Evolutionary Computation Conference, pp. 1-5, 2000

[83] K. DeJong, "Learning with genetic algorithms: an overview," Mach. Lang., vol. 3, no. 2-3, pp. 121-138, 1988.

[84] H. G. Cobb and J. J. Grefenstette, "Genetic Algorithms for Tracking Changing Environments," in Proceedings of International Conference on Genetic Algorithms, 1993, pp. 523-530, 657576.

[85] J. D. Schaffer, R. A. Caruana, L. J. Eshelman, and R. Das, "A study of control parameters affecting online performance of genetic algorithms for function optimization," presented at the Proceedings of the international conference on Genetic algorithms, 1989.

[86] V. P. Patil and D. D. Pawar, "The optimal crossover or mutation rates in Genetic algorithm : A Review," International Journal of Applied Engineering and Technology, vol. 5, pp. 38-41, 2015.

[87] H. M, \#252, and hlenbein, "Parallel Genetic Algorithms Population Genetics and Combinatorial Optimization," in Proceedings of International Conference on Genetic Algorithms, 1989, pp. 416-421, 657252.

[88] S. A. Jolly, V. Garousi, and M. M. Eskandar, "Automated Unit Testing of a SCADA Control Software: An Industrial Case Study based on Action Research," in IEEE International Conference on Software Testing, Verification and Validation (ICST), 2012, pp. 400-409.

[89] P. R. C. Wohlin, M. Höst, M. C. Ohlsson, B. Regnell, and A. Wesslén, "Experimentation in Software Engineering: An Introduction," 2000.

[90] C. Wohlin, P. Runeson, M. Höst, M. C. Ohlsson, B. Regnell, and A. Wesslén, Experimentation in Software Engineering: An Introduction. Kluwer Academic Publishers, 2000. 